\title{
The modified split generalized equilibrium problem for quasi-nonexpansive mappings and applications
}

Kanyarat Cheawchan ${ }^{1}$ and Atid Kangtunyakarn ${ }^{1 *}$

"Correspondence:
beawrock@hotmail.com
'Department of Mathematics,
Faculty of Science, King Mongkut's
Institute of Technology Ladkrabang,
Bangkok, Thailand

"Correspondence:

beawrock@hotmail.com

Faculty of Science, King Mongkut's

Bangkok, Thailand

\begin{abstract}
In this paper, we introduce a new problem, the modified split generalized equilibrium problem, which extends the generalized equilibrium problem, the split equilibrium problem and the split variational inequality problem. We introduce a new method of an iterative scheme $\left\{x_{n}\right\}$ for finding a common element of the set of solutions of variational inequality problems and the set of common fixed points of a finite family of quasi-nonexpansive mappings and the set of solutions of the modified split generalized equilibrium problem without assuming a demicloseness condition and $T_{\omega}:=(1-\omega) l+\omega T$, where $T$ is a quasi-nonexpansive mapping and $\omega \in\left(0, \frac{1}{2}\right) ;$ a difficult proof in the framework of Hilbert space. In addition, we give a numerical example to support our main result.
\end{abstract}

MSC: 47B40; 47H10;47J20

Keywords: The modified split generalized equilibrium problem; Quasi-nonexpansive mapping; Variational Inequality problem; Fixed Point problem

\section{Introduction}

Let $C$ be a nonempty closed convex subset of a real Hilbert space $H$. The set of fixed points of $T$ is denoted by $F(T)$. The mapping $T: C \rightarrow C$ is said to be quasi-nonexpansive if

$$
\|T x-p\| \leq\|x-p\|
$$

for all $x \in C$ and $p \in F(T)$.

Definition 1.1 ([1]) Let $T: H \rightarrow H$. Then the following are equivalent:

1. $T$ is firmly nonexpansive,

2. $\|T x-T y\|^{2} \leq\langle x-y, T x-T y\rangle, \forall x, y \in H$,

3. $\langle T x-T y,(I-T) x-(I-T) y\rangle \geq 0, \forall x, y \in H$.

Let $A: C \rightarrow H$ be a mapping. The variational inequality is to find a point $u \in C$ such that

$$
\langle A u, v-u\rangle \geq 0,
$$

(c) The Author(s) 2018. This article is distributed under the terms of the Creative Commons Attribution 4.0 International License (http://creativecommons.org/licenses/by/4.0/), which permits unrestricted use, distribution, and reproduction in any medium, provided you give appropriate credit to the original author(s) and the source, provide a link to the Creative Commons license, and indicate if changes were made. 
for all $v \in C$. The set of solutions of (1.1) is denoted by $V I(C, A)$. A mapping $A: C \rightarrow H$ is called $\alpha$-inverse strongly monotone if there exists a positive real number $\alpha>0$ such that

$$
\langle A x-A y, x-y\rangle \geq \alpha\|A x-A y\|^{2},
$$

for all $x, y \in C$. They have been investigated in the literature; see, for example, [2, 3]. Let $F$ be a bifunction of $C \times C$ into $\mathbb{R}$, where $\mathbb{R}$ is the set of real numbers. The equilibrium problem for $F: C \times C \rightarrow \mathbb{R}$ is to find $x \in C$ such that

$$
F(x, y) \geq 0, \quad \forall y \in C
$$

The set of solutions of (1.2) is denoted by $E P(F)$. Equilibrium problems were introduced by [4] in 1994 and included many well-known problems such as variational inequality, optimization problem, nonexpansive mapping and fixed point problem; see, for example, [5-8].

Let $F$ be a function of $C \times C$ into $\mathbb{R}$ and let $f: H \rightarrow H$ be a mapping. The generalized equilibrium problem is to find $x \in C$ such that

$$
F(x, y)+\langle f(x), y-x\rangle \geq 0
$$

for all $y \in C$. The set of solutions of (1.3) is denoted by $E P(F, f)$. When $f \equiv 0, E P(F, f)$ is denoted by $E P(F)$ and $F \equiv 0, E P(F, f)$ is denoted by $V I(C, f)$.

Throughout this section, let $H_{1}, H_{2}$ be real Hilbert spaces and let $C, Q$ be nonempty closed convex subsets of real Hilbert spaces $H_{1}$ and $H_{2}$, respectively. Let $A: H_{1} \rightarrow H_{2}$ be a bounded linear operator.

In 1994, Censor and Elfving [9] introduced the split feasibility problem (in short, SFP) which is to find a point $x \in C$ such that $A x \in Q$. The set of all solutions of split feasibility problem is denoted by $\varphi=\{x \in C: A x \in Q\}$.

To solve the SFP, Byrne [10] introduced CQ algorithm whose sequence $\left\{x_{n}\right\}$ is generated by

$$
x_{n+1}=P_{C_{1}}\left(x_{n}-\gamma A^{*}\left(I-P_{C_{2}}\right) A x_{n}\right),
$$

where the initial $x_{0} \in H_{1}$ and $\gamma \in(0,2 / L), L$ is the spectral radius of the operator $A^{*} A$ and $A^{*}$ is the adjoint of $A$. Then the $C Q$ algorithm converges to a solution of the SFP, whenever solutions exist. If there are no solutions of the SFP, the $C Q$ algorithm converges to a minimizer of the function

$$
\frac{1}{2}\left\|\left(I-P_{C_{2}}\right) A x\right\|^{2}
$$

whenever such minimizers exist.

Let $U: H_{1} \rightarrow H_{1}$ and $T: H_{2} \rightarrow H_{2}$ be two nonlinear operators. The split common fixed points problem (SCFPP) $[11,12]$ is to find a point $x^{*}$ such that

$$
x^{*} \in F(U) \text { and } A x^{*} \in F(T) .
$$


The solution set of SCFPP is denoted by $\Phi=\left\{p^{*} \in F(U): A p^{*} \in F(T)\right\}$. The split common fixed point problem is a generalization of the split feasibility problem.

In 2017, Wang [13] introduced a new method for solving SCFPP as follows:

$$
x_{n+1}=x_{n}-\rho_{n}\left((I-U) x_{n}+A^{*}(I-T) A x_{n}\right),
$$

where $\rho_{n} \subset(0, \infty)$ is chosen such that

$$
\rho_{n}=\frac{\left\|(I-U) x_{n}\right\|^{2}+\left\|(I-T) A x_{n}\right\|^{2}}{\left\|(I-U) x_{n}+A^{*}(I-T) A x_{n}\right\|^{2}}
$$

and $U$ and $T$ are firmly quasi-nonexpansive mappings. Then the sequence $\left\{x_{n}\right\}$ converges weakly to $z$, where $z=\lim _{n \rightarrow \infty} P_{\Phi} x_{n}$.

Censor et al. [11, 14] introduced the prototypical split inverse problem (SIP) which is a generalization of the split common fixed points problem. In this, there are given two vector spaces $X$ and $Y$ and a linear operator $A: X \rightarrow Y$. In addition, two inverse problems are involved. The first one, denoted $\mathrm{IP}_{1}$, is formulated in the space $X$ and the second one, denoted $\mathrm{IP}_{2}$, is formulated in the space $Y$. Given these data, the split inverse problem is formulated as follows:

$$
\text { find a point } x^{*} \in X \text { that solves } \mathrm{IP}_{1} \text {, }
$$

and such that

$$
\text { find a point } y^{*} \in Y \text { that solves } \mathrm{IP}_{2} \text {. }
$$

This problem is used in many modeling arising in sensor networks, radiation therapy treatment planning, color imaging, etc.

The split equilibrium problem (SEP) [12] is to find $\widehat{x} \in C$ such that

$$
F_{1}(\widehat{x}, x) \geq 0, \quad \forall x \in C,
$$

and such that

$$
\widehat{y}=A \widehat{x} \in Q \quad \text { solves } F_{2}(\widehat{y}, y) \geq 0, \quad \forall y \in Q,
$$

where $F_{1}: C \times C \rightarrow \mathbb{R}$ and $F_{2}: Q \times Q \rightarrow \mathbb{R}$ be nonlinear bifunctions. If we consider only problem (1.7), it is the equilibrium problem and we denoted its solution set by $E P\left(F_{1}\right)$. The solution set of SEP is denoted by $\Gamma=\left\{\widehat{p} \in E P\left(F_{1}\right): A \widehat{p} \in E P\left(F_{2}\right)\right\}$. SEP is reduced to $E P(F)$, where $H_{1} \equiv H_{2}, F_{1} \equiv F_{2}$ and $A \equiv I . E P(F)$ is an unifying model for several problems arising in physics, engineering, science, optimization, economics, etc.

The split variational inequality problems (in short, SVIP) were introduced and studied by Cencor et al. [11]: find $\bar{x} \in C$ such that

$$
\left\langle f_{1}(\bar{x}), x-\bar{x}\right\rangle \geq 0, \quad \forall x \in C,
$$


and such that

$$
\bar{y}=A \bar{x} \in Q \quad \text { solves }\left\langle f_{2}(\bar{y}), y-\bar{y}\right\rangle \geq 0, \quad \forall y \in Q,
$$

where $f_{1}: C \rightarrow H_{1}$ and $f_{2}: Q \rightarrow H_{2}$ are nonlinear mappings. The solution set of SVIP is denoted by $\Psi=\left\{\bar{p} \in V I\left(C, f_{1}\right): A \bar{p} \in V I\left(Q, f_{2}\right)\right\}$. The split variational inequality problems have already been studied and used in practice as a model in intensity-modulated radiation therapy (IMRT) treatment planning; see, for example, [15] and the modeling of many inverse problems arising for phase retrieval and other real-world problems; for instance, in sensor networks in computerized tomography and data compression; see, for example, $[16,17]$.

By investigating SEP and SVIP, we introduce the modified split generalized equilibrium problem (MSGEP) which is to find $x^{*} \in C$ such that

$$
F_{1}\left(x^{*}, x\right)+\left\langle f_{1}\left(x^{*}\right), x-x^{*}\right\rangle \geq 0, \quad \forall x \in C,
$$

and such that

$$
y^{*}=A x^{*} \in Q \quad \text { solves } F_{2}\left(y^{*}, y\right)+\left\langle f_{2}\left(y^{*}\right), y-y^{*}\right\rangle \geq 0, \quad \forall y \in Q
$$

where $F_{1}: C \times C \rightarrow \mathbb{R}$ and $F_{2}: Q \times Q \rightarrow \mathbb{R}$ are nonlinear bifunctions and $f_{1}: C \rightarrow H_{1}$ and $f_{2}: Q \rightarrow H_{2}$ are nonlinear mappings. The solution set of MSGEP is denoted by $\Omega=\left\{p^{*} \in\right.$ $\left.E P\left(F_{1}, f_{1}\right): A p^{*} \in E P\left(F_{2}, f_{2}\right)\right\}$.

\section{Remark 1.1}

1. If we put $f_{1} \equiv f_{2} \equiv 0$ in MSGEP then the MSGEP is reduced to SEP.

2. If we put $F_{1} \equiv F_{2} \equiv 0$ in MSGEP then the MSGEP is reduced to SVIP.

3. In the case of bifunctions $F_{1}$ and $F_{2}$ are according to (A1)-(A4). From (1.11), (1.12) and Lemma 2.2, we have $x^{*} \in F\left(T_{r}^{F_{1}}\left(I-r f_{1}\right)\right)$ and $A x^{*} \in F\left(T_{s}^{F_{2}}\left(I-s f_{2}\right)\right)$, for all $r, s>0$. So, MSGEP can be viewed as SCFPP.

MSGEP is a generalization of the generalized equilibrium problem, the split equilibrium problem and the split variational inequality problem. So, this problem can be used in sensor networks, data compression, practice as a model in intensity-modulated radiation therapy (IMRT) treatment planning, robustness to marginal changes and equilibrium stability etc.

Example 1.2 Let $H_{1}=[0,6], H_{2}=[0,18], C=[2,5]$ and $Q=[6,10]$. Let $A: H_{1} \rightarrow H_{2}$ be defined by $A x=3 x$ for all $x \in H_{1}$. Let the mapping $F_{1}: C \times C \rightarrow \mathbb{R}$ be defined by

$$
F_{1}\left(x^{*}, x\right)=-\left(x^{*}-2\right)^{2}+(x-2)^{2}, \quad \forall x, y \in C,
$$

and $F_{2}: Q \times Q \rightarrow \mathbb{R}$ be defined by

$$
F_{2}\left(y^{*}, y\right)=-\left(y^{*}-6\right)^{2}+(y-6)^{2}, \quad \forall x, y \in Q .
$$


Let the mapping $f_{1}: C \rightarrow H_{1}$ be defined by $f_{1} x=\frac{x-2}{9}, \forall x \in C$ and the mapping $f_{2}: Q \rightarrow H_{2}$ be defined by $f_{2} x=\frac{x-6}{7}, \forall x \in Q$.

Then $2 \in \Omega$. Therefore 2 is a solution of MSGEP.

In 2012, Tain and Jin [18] introduced iterative algorithms involving a quasi-nonexpansive mapping. They generated the iterative as follows:

$$
x_{n+1}=\alpha_{n} \gamma f\left(x_{n}\right)+\left(I-\alpha_{n} A\right) T_{\omega} x_{n},
$$

where $A$ is a bounded linear operator on $H, T$ is a quasi-nonexpansive mapping on $H, f$ is a contraction with coefficient $a$ under suitable conditions of the parameters $\alpha_{n}, \gamma$ and $\omega$. By assuming $\omega \in\left(0, \frac{1}{2}\right), T_{\omega}:=(1-\omega) I+\omega T$ and $T$ is demiclosed on $H$.

Motivated by SFP and SVIP, we introduced a new problem, the modified split generalized equilibrium problem, which extends the generalized equilibrium problem, the split equilibrium problem and the split variational inequality problem. Many authors proved strong convergence theorem involving a quasi-nonexpansive mapping $T$ by assuming $T_{\omega}:=(1-\omega) I+\omega T$ and $T$ is demiclosed on $H$; a difficult proof. Motivated by [19], we introduced Remark 2.5 and $[11,12]$ and [18], we introduce a new method of iterative scheme $\left\{x_{n}\right\}$ for finding a common element of the set of solutions of variational inequality problems and the set of common fixed points of a finite family of quasi-nonexpansive mappings and the set of solutions of the modified split generalized equilibrium problem without the condition above in the framework of a Hilbert space.

\section{Preliminaries}

Let $H$ be a real Hilbert space with inner product $\langle\cdot, \cdot\rangle$ and norm $\|\cdot\|$. Throughout this paper, we use the notations of weak and strong convergence by " $\rightarrow$ " and " $\rightarrow$ ", respectively. Recall that $H$ satisfies Opial's condition [20], i.e., for any sequence $\left\{x_{n}\right\}$ with $x_{n} \rightarrow x$, the inequality $\lim _{n \rightarrow \infty} \inf \left\|x_{n}-x\right\|<\lim _{n \rightarrow \infty} \inf \left\|x_{n}-y\right\|$, holds for every $y \in H$ with $y \neq x$.

For solving the equilibrium problem, we assume that the bifunction $F: C \times C \rightarrow \mathbb{R}$ satisfy the following conditions:

(A1) $F(x, x)=0$ for all $x \in C$,

(A2) $F$ is monotone, i.e., $F(x, y)+F(y, x) \leq 0$ for all $x, y \in C$,

(A3) for each $x, y, z \in C, \lim _{t \downarrow 0} F(t z+(1-t) x, y) \leq F(x, y)$,

(A4) for each $x \in C, y \mapsto F(x, y)$ is convex and lower semicontinuous.

Lemma 2.1 ([4]) Let $C$ be a nonempty closed convex subset of $H$ and let $F$ be a bifunction of $C \times C$ into $\mathbb{R}$ satisfying (A1)-(A4). Let $r>0$ and $x \in H$. Then there exists $z \in C$ such that

$$
F(z, y)+\frac{1}{r}\langle y-z, z-x\rangle \geq 0, \quad \forall y \in C .
$$

Lemma 2.2 ([21]) Assume that $F: C \times C \rightarrow \mathbb{R}$ satisfies (A1)-(A4). For $r>0$, define a mapping $T_{r}: H \rightarrow C$ as follows:

$$
T_{r}(x)=\left\{z \in C: F(z, y)+\frac{1}{r}\langle y-z, z-x\rangle \geq 0, \forall y \in C\right\}
$$

for all $x \in H$. Then the following hold: 
(1) $T_{r}$ is single-valued,

(2) $T_{r}$ is firmly nonexpansive, i.e., for any $x, y \in H$,

$$
\left\|T_{r}(x)-T_{r}(y)\right\|^{2} \leq\left\langle T_{r}(x)-T_{r}(y), x-y\right\rangle
$$

(3) $F\left(T_{r}\right)=E P(F)$,

(4) $E P(F)$ is closed and convex.

Lemma 2.3 ([22]) Let $H$ be a real Hilbert space, let $C$ be a nonempty closed convex subset of $H$ and let $A$ be a mapping of $C$ into $H$. Let $u \in C$. Then, for $\lambda>0$,

$$
u=P_{C}(I-\lambda A) u \quad \Leftrightarrow \quad u \in V I(C, A),
$$

where $P_{C}$ is the metric projection of $H$ onto $C$.

Lemma 2.4 Let $C$ be a nonempty closed convex subset of a real Hilbert space $H$. Let $\left\{T_{i}\right\}_{i=1}^{N}$ be a finite family of quasi-nonexpansive mappings of $C$ into $H$ with $\bigcap_{i=1}^{N} F\left(T_{i}\right) \neq \emptyset$ and let $0<a_{i}<1$ with $\sum_{i=1}^{N} a_{i}=1$. Then

$$
\bigcap_{i=1}^{N} F\left(T_{i}\right)=V I\left(C, \sum_{i=1}^{N} a_{i}\left(I-T_{i}\right)\right) .
$$

Proof In this lemma, we show that $\bigcap_{i=1}^{N} F\left(T_{i}\right)=\bigcap_{i=1}^{N} V I\left(C, I-T_{i}\right)$ and $\bigcap_{i=1}^{N} V I\left(C, I-T_{i}\right)=$ $V I\left(C, \sum_{i=1}^{N} a_{i}\left(I-T_{i}\right)\right)$. Lastly, we have

$$
\bigcap_{i=1}^{N} F\left(T_{i}\right)=V I\left(C, \sum_{i=1}^{N} a_{i}\left(I-T_{i}\right)\right) .
$$

To start with, it is easy to see that $\bigcap_{i=1}^{N} F\left(T_{i}\right) \subseteq \bigcap_{i=1}^{N} V I\left(C, I-T_{i}\right)$. Next, we show that $\bigcap_{i=1}^{N} V I\left(C, I-T_{i}\right) \subseteq \bigcap_{i=1}^{N} F\left(T_{i}\right)$. Let $u \in \bigcap_{i=1}^{N} V I\left(C, I-T_{i}\right)$ and $\bigcap_{i=1}^{N} F\left(T_{i}\right) \neq \emptyset$. So, we get $u \in V I\left(C, I-T_{i}\right), \forall i=1,2, \ldots, N$. We may write

$$
\left\langle u-v,\left(I-T_{i}\right) u\right\rangle \leq 0, \quad \forall v \in C
$$

There exists $v^{*} \in C$ such that $v^{*}=T_{i} v^{*}, \forall i=1,2, \ldots, N$. Since $T_{i}$ is a quasi-nonexpansive mapping, $\forall i=1,2, \ldots, N$, it follows that

$$
\begin{aligned}
\left\|T_{i} u-v^{*}\right\|^{2} & =\left\|\left(u-v^{*}\right)-\left(I-T_{i}\right) u\right\|^{2} \\
& =\left\|u-v^{*}\right\|^{2}-2\left\langle u-v^{*},\left(I-T_{i}\right) u\right\rangle+\left\|\left(I-T_{i}\right) u\right\|^{2} \\
& \leq\left\|u-v^{*}\right\|^{2} .
\end{aligned}
$$

By using (2.1) and (2.2), we conclude that

$$
\left\|\left(I-T_{i}\right) u\right\|^{2} \leq 2\left\langle u-v^{*},\left(I-T_{i}\right) u\right\rangle \leq 0 .
$$


It implies that $u \in \bigcap_{i=1}^{N} F\left(T_{i}\right)$. Therefore $\bigcap_{i=1}^{N} V I\left(C, I-T_{i}\right) \subseteq \bigcap_{i=1}^{N} F\left(T_{i}\right)$. Hence

$$
\bigcap_{i=1}^{N} F\left(T_{i}\right)=\bigcap_{i=1}^{N} V I\left(C, I-T_{i}\right)
$$

After that, we show $\bigcap_{i=1}^{N} \operatorname{VI}\left(C, I-T_{i}\right)=V I\left(C, \sum_{i=1}^{N} a_{i}\left(I-T_{i}\right)\right)$ where $0<a_{i}<1$ and $\sum_{i=1}^{N} a_{i}=1$. Observe that

$$
\begin{aligned}
u & \in \bigcap_{i=1}^{N} V I\left(C, I-T_{i}\right) \\
& \Leftrightarrow \quad u \in V I\left(C, I-T_{i}\right), \quad \forall i=1,2, \ldots, N \\
& \Leftrightarrow \quad\left\langle\left(I-T_{i}\right) u, v-u\right\rangle \geq 0, \quad \forall v \in C \text { and } \forall i=1,2, \ldots, N \\
& \Leftrightarrow \quad \sum_{i=1}^{N} a_{i}\left(\left(I-T_{i}\right) u, v-u\right\rangle \geq 0, \quad \forall v \in C \\
& \Leftrightarrow \quad\left\langle\sum_{i=1}^{N} a_{i}\left(I-T_{i}\right) u, v-u\right\rangle \geq 0, \quad \forall v \in C \\
& \Leftrightarrow \quad u \in V I\left(C, \sum_{i=1}^{N} a_{i}\left(I-T_{i}\right)\right) .
\end{aligned}
$$

Therefore $\bigcap_{i=1}^{N} V I\left(C, I-T_{i}\right)=V I\left(C, \sum_{i=1}^{N} a_{i}\left(I-T_{i}\right)\right)$. Hence $\bigcap_{i=1}^{N} F\left(T_{i}\right)=V I\left(C, \sum_{i=1}^{N} a_{i}(I-\right.$ $\left.T_{i}\right)$ ).

Remark 2.5 From Lemma 2.3 and Lemma 2.4, we have

$$
\bigcap_{i=1}^{N} F\left(T_{i}\right)=V I\left(C, \sum_{i=1}^{N} a_{i}\left(I-T_{i}\right)\right)=F\left(P_{C}\left(I-\lambda\left(\sum_{i=1}^{N} a_{i}\left(I-T_{i}\right)\right)\right)\right),
$$

for all $\lambda>0$ and $0<a_{i}<1$ with $\sum_{i=1}^{N} a_{i}=1$.

Lemma 2.6 ([23]) Let $\left\{s_{n}\right\}$ be a sequence of nonnegative real numbers satisfying

$$
s_{n+1} \leq\left(1-\alpha_{n}\right) s_{n}+\delta_{n}, \quad \forall n \geq 0,
$$

where $\left\{\alpha_{n}\right\}$ is a sequence in $(0,1)$ and $\left\{\delta_{n}\right\}$ is a sequence such that
(1) $\sum_{n=1}^{\infty} \alpha_{n}=\infty$
(2) $\quad \limsup _{n \rightarrow \infty} \frac{\delta_{n}}{\alpha_{n}} \leq 0 \quad$ or $\quad \sum_{n=1}^{\infty}\left|\delta_{n}\right|<\infty$.

Then $\lim _{n \rightarrow \infty} s_{n}=0$.

\section{Main results}

Lemma 3.1 Let $C$ and $Q$ be nonempty closed convex subsets of a real Hilbert spaces $H_{1}$ and $H_{2}$, respectively. Let $A: H_{1} \rightarrow H_{2}$ be a bounded linear operator. Let $F_{1}: C \times C \rightarrow \mathbb{R}$ and 
$F_{2}: Q \times Q \rightarrow \mathbb{R}$ be the bifunctions satisfying (A1)-(A4). Let $f_{1}: H_{1} \rightarrow H_{1}$ be a $\rho$-inverse strongly monotone mapping and $f_{2}: H_{2} \rightarrow H_{2}$ be a firmly nonexpansive mapping. Then

1. $T_{r}^{F_{1}}\left(I-r f_{1}\right)$ and $T_{s}^{F_{2}}\left(I-s f_{2}\right)$ are nonexpansive mapping,

2.

$$
\begin{aligned}
\| T_{r}^{F_{1}} & \left(I-r f_{1}\right)\left(p+\gamma A^{*}\left(T_{s}^{F_{2}}\left(I-s f_{2}\right)-I\right) A p\right) \\
& \quad-T_{r}^{F_{1}}\left(I-r f_{1}\right)\left(q+\gamma A^{*}\left(T_{s}^{F_{2}}\left(I-s f_{2}\right)-I\right) A q\right) \|^{2} \\
\leq & \|p-q\|^{2}+\gamma(\gamma L-1)\left\|\left(T_{s}^{F_{2}}\left(I-s f_{2}\right)-I\right) A p-\left(T_{s}^{F_{2}}\left(I-s f_{2}\right)-I\right) A q\right\|^{2},
\end{aligned}
$$

for all $p, q \in C$, where $r \in(0,2 \rho), s \in(0,1), \gamma \in(0,1 / L), L$ is the spectral radius of the operator $A^{*} A$ and $A^{*}$ is the adjoint of $A, T_{r}^{F_{1}}: H_{1} \rightarrow C$ defined by

$$
T_{r}^{F_{1}}(x)=\left\{z \in C: F_{1}(z, y)+\frac{1}{r}\langle y-z, z-x\rangle \geq 0, \forall y \in C\right\}
$$

for all $x \in H_{1}$ and $T_{s}^{F_{2}}: H_{2} \rightarrow Q$ defined by

$$
T_{s}^{F_{2}}(\bar{x})=\left\{\bar{z} \in Q: F_{2}(\bar{z}, y)+\frac{1}{s}\langle y-\bar{z}, \bar{z}-\bar{x}\rangle \geq 0, \forall y \in Q\right\},
$$

for all $\bar{x} \in H_{2}$.

Proof Let $p, q \in C$. First, we show 1 is true. Since $f_{1}$ is a $\rho$-inverse strongly monotone mapping and $r \in(0,2 \rho)$, we obtain

$$
\begin{aligned}
\left\|T_{r}^{F_{1}}\left(I-r f_{1}\right) p-T_{r}^{F_{1}}\left(I-r f_{1}\right) q\right\|^{2} & \leq\|p-q\|^{2}-2 r\left\langle p-q, f_{1} p-f_{1} q\right\rangle+r^{2}\left\|f_{1} p-f_{1} q\right\|^{2} \\
& \leq\|p-q\|^{2}+r(r-2 \rho)\left\|f_{1} p-f_{1} q\right\|^{2} \\
& \leq\|p-q\|^{2} .
\end{aligned}
$$

Thus $T_{r}^{F_{1}}\left(I-r f_{1}\right)$ is a nonexpansive mapping. Since $f_{2}$ is a firmly nonexpansive mapping and $s \in(0,1)$, we get

$$
\begin{aligned}
\left\|T_{s}^{F_{2}}\left(I-s f_{2}\right) \bar{p}-T_{s}^{F_{2}}\left(I-s f_{2}\right) \bar{q}\right\|^{2} & \leq\|\bar{p}-\bar{q}\|^{2}-2 s\left\langle\bar{p}-\bar{q}, f_{2} \bar{p}-f_{2} \bar{q}\right\rangle+s^{2}\left\|f_{2} \bar{p}-f_{2} \bar{q}\right\|^{2} \\
& \leq\|\bar{p}-\bar{q}\|^{2}-s(2-s)\left\|f_{2} \bar{p}-f_{2} \bar{q}\right\|^{2} \\
& \leq\|\bar{p}-\bar{q}\|^{2}
\end{aligned}
$$

for all $\bar{p}, \bar{q} \in Q$. Therefore $T_{s}^{F_{2}}\left(I-s f_{2}\right)$ is a nonexpansive mapping.

Next, we show 2 is true. From Lemma 3.1(1), we have

$$
\begin{aligned}
& \| T_{r}^{F_{1}}\left(I-r f_{1}\right)\left(p+\gamma A^{*}\left(T_{s}^{F_{2}}\left(I-s f_{2}\right)-I\right) A p\right) \\
& \quad-T_{r}^{F_{1}}\left(I-r f_{1}\right)\left(q+\gamma A^{*}\left(T_{s}^{F_{2}}\left(I-s f_{2}\right)-I\right) A q\right) \|^{2} \\
& \leq\left\|(p-q)+\gamma\left(A^{*}\left(T_{s}^{F_{2}}\left(I-s f_{2}\right)-I\right) A p-A^{*}\left(T_{s}^{F_{2}}\left(I-s f_{2}\right)-I\right) A q\right)\right\|^{2} \\
& \leq\|p-q\|^{2}+2 \gamma\left(A p-A q,\left(T_{s}^{F_{2}}\left(I-s f_{2}\right)-I\right) A p-\left(T_{s}^{F_{2}}\left(I-s f_{2}\right)-I\right) A q\right\rangle \\
&+\gamma^{2} L\left\|\left(T_{s}^{F_{2}}\left(I-s f_{2}\right)-I\right) A p-\left(T_{s}^{F_{2}}\left(I-s f_{2}\right)-I\right) A q\right\|^{2} .
\end{aligned}
$$


From the property of $T_{s}^{F_{2}}$, we get

$$
\begin{aligned}
\|(I- & \left.s f_{2}\right) A p-\left(I-s f_{2}\right) A q \|^{2} \\
\geq & \left\|T_{s}^{F_{2}}\left(I-s f_{2}\right) A p-T_{s}^{F_{2}}\left(I-s f_{2}\right) A q-(A p-A q)+(A p-A q)\right\|^{2} \\
= & \left\|\left(T_{s}^{F_{2}}\left(I-s f_{2}\right)-I\right) A p-\left(T_{s}^{F_{2}}\left(I-s f_{2}\right)-I\right) A q\right\|^{2} \\
& +2\left\langle\left(T_{s}^{F_{2}}\left(I-s f_{2}\right)-I\right) A p-\left(T_{s}^{F_{2}}\left(I-s f_{2}\right)-I\right) A q, A p-A q\right\rangle \\
& +\|A p-A q\|^{2} .
\end{aligned}
$$

We have

$$
\begin{aligned}
\|(I- & \left.s f_{2}\right) A p-\left(I-s f_{2}\right) A q \|^{2} \\
= & \|A p-A q\|^{2}-2 s\left\langle A p-A q, f_{2} A p-f_{2} A q\right\rangle \\
& +s^{2}\left\|f_{2} A p-f_{2} A q\right\|^{2} .
\end{aligned}
$$

From (3.2), (3.3) and the property of firmly nonexpansive mapping, we get

$$
\begin{gathered}
2\left\langle\left(T_{s}^{F_{2}}\left(I-s f_{2}\right)-I\right) A p-\left(T_{s}^{F_{2}}\left(I-s f_{2}\right)-I\right) A q, A p-A q\right\rangle \\
\leq-\left\|\left(T_{s}^{F_{2}}\left(I-s f_{2}\right)-I\right) A p-\left(T_{s}^{F_{2}}\left(I-s f_{2}\right)-I\right) A q\right\|^{2} \\
\quad-2 s\left\langle A p-A q, f_{2} A p-f_{2} A q\right\rangle+s^{2}\left\|f_{2} A p-f_{2} A q\right\|^{2} \\
\leq-\left\|\left(T_{s}^{F_{2}}\left(I-s f_{2}\right)-I\right) A p-\left(T_{s}^{F_{2}}\left(I-s f_{2}\right)-I\right) A q\right\|^{2} .
\end{gathered}
$$

That is,

$$
\begin{gathered}
2 \gamma\left\langle\left(T_{s}^{F_{2}}\left(I-s f_{2}\right)-I\right) A p-\left(T_{s}^{F_{2}}\left(I-s f_{2}\right)-I\right) A q, A p-A q\right\rangle \\
\leq-\gamma\left\|\left(T_{s}^{F_{2}}\left(I-s f_{2}\right)-I\right) A p-\left(T_{s}^{F_{2}}\left(I-s f_{2}\right)-I\right) A q\right\|^{2} .
\end{gathered}
$$

Substituting (3.4) in (3.1), we obtain

$$
\begin{aligned}
\| T_{r}^{F_{1}}\left(I-r f_{1}\right)\left(p+\gamma A^{*}\left(T_{s}^{F_{2}}\left(I-s f_{2}\right)-I\right) A p\right) \\
\quad-T_{r}^{F_{1}}\left(I-r f_{1}\right)\left(q+\gamma A^{*}\left(T_{s}^{F_{2}}\left(I-s f_{2}\right)-I\right) A q\right) \|^{2} \\
\leq\|p-q\|^{2}-\gamma\left\|\left(T_{s}^{F_{2}}\left(I-s f_{2}\right)-I\right) A p-\left(T_{s}^{F_{2}}\left(I-s f_{2}\right)-I\right) A q\right\|^{2} \\
\quad+\gamma^{2} L\left\|\left(T_{s}^{F_{2}}\left(I-s f_{2}\right)-I\right) A p-\left(T_{s}^{F_{2}}\left(I-s f_{2}\right)-I\right) A q\right\|^{2} \\
=\|p-q\|^{2}+\gamma(\gamma L-1)\left\|\left(T_{s}^{F_{2}}\left(I-s f_{2}\right)-I\right) A p-\left(T_{s}^{F_{2}}\left(I-s f_{2}\right)-I\right) A q\right\|^{2} .
\end{aligned}
$$

Lemma 3.2 Let $C$ be a nonempty closed convex subset of a real Hilbert space $H$ and let $T: C \rightarrow C$ be a quasi-nonexpansive mapping with $F(T) \neq \emptyset$. Then

$$
\|(I-T) x\|^{2} \leq 2\langle x-z,(I-T) x\rangle, \quad \forall x \in C
$$


Proof Let $x \in C$ and $z \in F(T)$. Since $T$ is a quasi-nonexpansive mapping, we get

$$
\begin{aligned}
\|T x-z\|^{2} & =\|(x-z)-(I-T) x\|^{2} \\
& =\|x-z\|^{2}-2\langle x-z,(I-T) x\rangle+\|(I-T) x\|^{2} \\
& \leq\|x-z\|^{2} .
\end{aligned}
$$

We can conclude that

$$
\|(I-T) x\|^{2} \leq 2|x-z,(I-T) x\rangle
$$

Lemma 3.3 Let C be a nonempty closed convex subset of a real Hilbert space H. Let $\left\{T_{i}\right\}_{i=1}^{N}$ be a finite family of quasi-nonexpansive mappings of $C$ into itself with $\bigcap_{i=1}^{N} F\left(T_{i}\right) \neq \emptyset$. Then

$$
\left\|P_{C}\left(I-\bar{\lambda}\left(\sum_{i=1}^{N} k_{i}\left(I-T_{i}\right)\right)\right) x-z\right\|^{2} \leq\|x-z\|^{2},
$$

for all $x \in C$, where $0<k_{i}<1$ with $\sum_{i=1}^{N} k_{i}=1$ and $0<\bar{\lambda}<1$.

Proof Let $x \in C$ and $z \in \bigcap_{i=1}^{N} F\left(T_{i}\right)$. From Remark 2.5 and $z \in \bigcap_{i=1}^{N} F\left(T_{i}\right)$, we have $z \in$ $F\left(P_{C}\left(I-\bar{\lambda}\left(\sum_{i=1}^{N} k_{i}\left(I-T_{i}\right)\right)\right)\right)$ and $z=T_{i} z, \forall i=1,2, \ldots, N$. Since $P_{C}$ is nonexpansive mapping, $0<\bar{\lambda}<1$ and Lemma 3.2, we have

$$
\begin{aligned}
& \left\|P_{C}\left(I-\bar{\lambda}\left(\sum_{i=1}^{N} k_{i}\left(I-T_{i}\right)\right)\right) x-z\right\|^{2} \\
& \quad=\left\|P_{C}\left(I-\bar{\lambda}\left(\sum_{i=1}^{N} k_{i}\left(I-T_{i}\right)\right)\right) x-P_{C}\left(I-\bar{\lambda}\left(\sum_{i=1}^{N} k_{i}\left(I-T_{i}\right)\right)\right) z\right\|^{2} \\
& \quad \leq\|x-z\|^{2}-2 \bar{\lambda} \sum_{i=1}^{N} k_{i}\left(x-z,\left(I-T_{i}\right) x\right\rangle+\bar{\lambda}^{2} \sum_{i=1}^{N} k_{i}\left\|\left(I-T_{i}\right) x\right\|^{2} \\
& \quad \leq\|x-z\|^{2}-\bar{\lambda} \sum_{i=1}^{N} k_{i}\left\|\left(I-T_{i}\right) x\right\|^{2}+\bar{\lambda}^{2} \sum_{i=1}^{N} k_{i}\left\|\left(I-T_{i}\right) x\right\|^{2} \\
& \quad \leq\|x-z\|^{2} .
\end{aligned}
$$

Next, we prove a strong convergence theorem for solving the modified split generalized equilibrium problem (MSGEP).

Theorem 3.4 Let $C$ and $Q$ be nonempty closed convex subsets of a real Hilbert spaces $H_{1}$ and $H_{2}$, respectively. Let $A: H_{1} \rightarrow H_{2}$ be a bounded linear operator. Let $D_{1}, D_{2}: C \rightarrow H_{1}$ be $\alpha, \beta$-inverse strongly monotone mappings, respectively. Let $F_{1}: C \times C \rightarrow \mathbb{R}$ and $F_{2}$ : $Q \times Q \rightarrow \mathbb{R}$ be the bifunctions satisfying (A1)-(A4). Let $\left\{T_{i}\right\}_{i=1}^{N}$ be a finite family of quasinonexpansive mappings of $C$ into itself with $\bigcap_{i=1}^{N} F\left(T_{i}\right) \neq \emptyset$. Let $f_{1}: H_{1} \rightarrow H_{1}$ be a $\rho$-inverse strongly monotone mapping and $f_{2}: H_{2} \rightarrow H_{2}$ be a firmly nonexpansive mapping. Assume $\mathcal{F}=V I\left(C, D_{1}\right) \cap V I\left(C, D_{2}\right) \cap \bigcap_{i=1}^{N} F\left(T_{i}\right) \cap \Omega \neq \emptyset$. For given $x_{1}, u \in C$ and let $\left\{x_{n}\right\},\left\{u_{n}\right\}$ and 
$\left\{y_{n}\right\}$ be sequences generated by

$$
\left\{\begin{array}{l}
u_{n}=T_{r}^{F_{1}}\left(I-r f_{1}\right)\left(x_{n}+\gamma A^{*}\left(T_{s}^{F_{2}}\left(I-s f_{2}\right)-I\right) A x_{n}\right), \\
y_{n}=P_{C}\left(I-d_{1} D_{1}\right)\left(a u_{n}+(1-a) P_{C}\left(I-d_{2} D_{2}\right) u_{n}\right), \\
x_{n+1}=\alpha_{n} u+\beta_{n} x_{n}+\gamma_{n} P_{C}\left(I-\lambda_{n}\left(\sum_{i=1}^{N} k_{i}\left(I-T_{i}\right)\right)\right) y_{n}, \quad \forall n \in \mathbb{N},
\end{array}\right.
$$

where $d_{1} \in(0,2 \alpha), d_{2} \in(0,2 \beta), r \in(0,2 \rho), s \in(0,1), a \in[0,1], 0<k_{i}<1$ with $\sum_{i=1}^{N} k_{i}=1$, $\gamma \in(0,1 / L), L$ is the spectral radius of the operator $A^{*} A$ and $A^{*}$ is the adjoint of $A$. Also $\left\{\alpha_{n}\right\}$, $\left\{\beta_{n}\right\},\left\{\gamma_{n}\right\}$ are sequences in $[0,1]$ with $\alpha_{n}+\beta_{n}+\gamma_{n}=1$ for all $n \in \mathbb{N}$. Suppose the following conditions hold:

(i) $\lim _{n \rightarrow \infty} \alpha_{n}=0$ and $\sum_{n=1}^{\infty} \alpha_{n}=\infty$,

(ii) $0<c \leq \beta_{n}, \gamma_{n} \leq d<1$ for some $c, d>0$ for all $n \geq 1$,

(iii) $\sum_{n=1}^{\infty} \lambda_{n}<\infty$ and $0<\lambda_{n}<1$,

(iv) $\sum_{n=1}^{\infty}\left|\alpha_{n+1}-\alpha_{n}\right|<\infty, \sum_{n=1}^{\infty}\left|\beta_{n+1}-\beta_{n}\right|<\infty$.

Then $\left\{x_{n}\right\},\left\{u_{n}\right\}$ and $\left\{y_{n}\right\}$ converge strongly to $z=P_{\mathcal{F}} u$.

Proof Let $x, y \in C$ and $z \in \mathcal{F}$. First, we show that $\left(I-d_{1} D_{1}\right)$ is a nonexpansive mapping. Since $D_{1}$ is an $\alpha$-inverse strongly monotone mapping, we obtain

$$
\begin{aligned}
\left\|\left(I-d_{1} D_{1}\right) x-\left(I-d_{1} D_{1}\right) y\right\|^{2} & =\|x-y\|^{2}-2 d_{1}\left\langle x-y, D_{1} x-D_{1} y\right\rangle+d_{1}^{2}\left\|D_{1} x-D_{1} y\right\|^{2} \\
& \leq\|x-y\|^{2}+d_{1}\left(d_{1}-2 \alpha\right)\left\|D_{1} x-D_{1} y\right\|^{2} \leq\|x-y\|^{2} .
\end{aligned}
$$

Thus $\left(I-d_{1} D_{1}\right)$ is a nonexpansive mapping. By using the same method as above, we see that $\left(I-d_{2} D_{2}\right)$ is a nonexpansive mapping. Since $f_{1}$ is a $\rho$-inverse strongly monotone mapping and $f_{2}$ is a firmly nonexpansive mapping. From Lemma 3.1(1), we have $\left(T_{r}^{F_{1}}\left(I-r f_{1}\right)\right)$ and $\left(T_{s}^{F_{2}}\left(I-s f_{2}\right)\right)$ are nonexpansive mappings. Since $z \in \bigcap_{i=1}^{N} F\left(T_{i}\right)$ and Lemma 3.3, we have

$$
\left\|P_{C}\left(I-\lambda_{n}\left(\sum_{i=1}^{N} k_{i}\left(I-T_{i}\right)\right)\right) y_{n}-z\right\|^{2} \leq\left\|y_{n}-z\right\|^{2} .
$$

Since $z \in V I\left(C, D_{1}\right)$ and $z \in V I\left(C, D_{2}\right)$ and using the property of $\left(I-d_{1} D_{1}\right)$ and $\left(I-d_{2} D_{2}\right)$, we get

$$
\begin{aligned}
\left\|y_{n}-z\right\|^{2} & =\left\|P_{C}\left(I-d_{1} D_{1}\right)\left(a u_{n}+(1-a) P_{C}\left(I-d_{2} D_{2}\right) u_{n}\right)-P_{C}\left(I-d_{1} D_{1}\right) z\right\|^{2} \\
& \leq a\left\|u_{n}-z\right\|^{2}+(1-a)\left\|P_{C}\left(I-d_{2} D_{2}\right) u_{n}-z\right\|^{2} \\
& \leq\left\|u_{n}-z\right\|^{2} .
\end{aligned}
$$

Since $z \in \Omega$, we have $z=T_{r}^{F_{1}}\left(I-r f_{1}\right) z$ and $A z=T_{s}^{F_{2}}\left(I-s f_{2}\right) A z$. From Lemma 3.1(2) and $\gamma \in(0,1 / L)$, we obtain

$$
\begin{aligned}
\left\|u_{n}-z\right\|^{2} & =\left\|T_{r}^{F_{1}}\left(I-r f_{1}\right)\left(x_{n}+\gamma A^{*}\left(T_{s}^{F_{2}}\left(I-s f_{2}\right)-I\right) A x_{n}\right)-T_{r}^{F_{1}}\left(I-r f_{1}\right) z\right\|^{2} \\
& \leq\left\|x_{n}-z\right\|^{2}+\gamma(L \gamma-1)\left\|\left(T_{s}^{F_{2}}\left(I-s f_{2}\right)-I\right) A x_{n}\right\|^{2} \\
& \leq\left\|x_{n}-z\right\|^{2} .
\end{aligned}
$$


Using the definition of $x_{n},(3.7),(3.9)$ and (3.11), we get

$$
\begin{aligned}
\left\|x_{n+1}-z\right\|= & \| \alpha_{n}(u-z)+\beta_{n}\left(x_{n}-z\right) \\
& +\gamma_{n}\left(P_{C}\left(I-\lambda_{n}\left(\sum_{i=1}^{N} k_{i}\left(I-T_{i}\right)\right)\right) y_{n}-z\right) \| \\
\leq & \alpha_{n}\|u-z\|+\beta_{n}\left\|x_{n}-z\right\|+\gamma_{n}\left\|y_{n}-z\right\| \\
\leq & \alpha_{n}\|u-z\|+\beta_{n}\left\|x_{n}-z\right\|+\gamma_{n}\left\|u_{n}-z\right\| \\
\leq & \alpha_{n}\|u-z\|+\left(1-\alpha_{n}\right)\left\|x_{n}-z\right\| .
\end{aligned}
$$

Using induction, we can conclude that

$$
\left\|x_{n}-z\right\| \leq \max \left\{\|u-z\|,\left\|x_{1}-z\right\|\right\}
$$

for all $n \geq 1$. This implies that the sequence $\left\{x_{n}\right\}$ is bounded and so are $\left\{y_{n}\right\}$ and $\left\{u_{n}\right\}$. From Lemma 3.1 (2) and $\gamma \in(0,1 / L)$, we obtain

$$
\begin{aligned}
\| u_{n}- & u_{n-1} \|^{2} \\
= & \| T_{r}^{F_{1}}\left(I-r f_{1}\right)\left(x_{n}+\gamma A^{*}\left(T_{s}^{F_{2}}\left(I-s f_{2}\right)-I\right) A x_{n}\right) \\
& \quad-T_{r}^{F_{1}}\left(I-r f_{1}\right)\left(x_{n-1}+\gamma A^{*}\left(T_{s}^{F_{2}}\left(I-s f_{2}\right)-I\right) A x_{n-1}\right) \|^{2} \\
\leq & \left\|x_{n}-x_{n-1}\right\|^{2}+\gamma(\gamma L-1)\left\|\left(T_{s}^{F_{2}}\left(I-s f_{2}\right)-I\right) A x_{n}-\left(T_{s}^{F_{2}}\left(I-s f_{2}\right)-I\right) A x_{n-1}\right\|^{2} \\
\leq & \left\|x_{n}-x_{n-1}\right\|^{2} .
\end{aligned}
$$

Next, we show that $\lim _{n \rightarrow \infty}\left\|x_{n+1}-x_{n}\right\|=0$. According to Eq. (3.12), we have

$$
\begin{aligned}
\left\|x_{n+1}-x_{n}\right\| & \|\left(\alpha_{n} u+\beta_{n} x_{n}+\gamma_{n} P_{C}\left(I-\lambda_{n}\left(\sum_{i=1}^{N} k_{i}\left(I-T_{i}\right)\right)\right) y_{n}\right) \\
& -\left(\alpha_{n-1} u+\beta_{n-1} x_{n-1}+\gamma_{n-1} P_{C}\left(I-\lambda_{n-1}\left(\sum_{i=1}^{N} k_{i}\left(I-T_{i}\right)\right)\right) y_{n-1}\right) \| \\
\leq & \left|\alpha_{n}-\alpha_{n-1}\right|\|u\|+\beta_{n}\left\|x_{n}-x_{n-1}\right\|+\left|\beta_{n}-\beta_{n-1}\right|\left\|x_{n-1}\right\|+\gamma_{n}\left\|y_{n}-y_{n-1}\right\| \\
& +\lambda_{n}\left\|\left(\sum_{i=1}^{N} k_{i}\left(I-T_{i}\right)\right) y_{n}-\left(\sum_{i=1}^{N} k_{i}\left(I-T_{i}\right)\right) y_{n-1}\right\| \\
& +\left|\lambda_{n}-\lambda_{n-1}\right|\left\|\left(\sum_{i=1}^{N} k_{i}\left(I-T_{i}\right)\right) y_{n-1}\right\| \\
& +\left|\gamma_{n}-\gamma_{n-1}\right|\left\|P_{C}\left(I-\lambda_{n-1}\left(\sum_{i=1}^{N} k_{i}\left(I-T_{i}\right)\right)\right) y_{n-1}\right\| \\
\leq & \left(1-\alpha_{n}\right)\left\|x_{n}-x_{n-1}\right\|+\left|\alpha_{n}-\alpha_{n-1}\right|\|u\|+\left|\beta_{n}-\beta_{n-1}\right|\left\|x_{n-1}\right\|
\end{aligned}
$$




$$
\begin{aligned}
& +\lambda_{n}\left\|\left(\sum_{i=1}^{N} k_{i}\left(I-T_{i}\right)\right) y_{n}-\left(\sum_{i=1}^{N} k_{i}\left(I-T_{i}\right)\right) y_{n-1}\right\| \\
& +\left|\lambda_{n}-\lambda_{n-1}\right|\left\|\left(\sum_{i=1}^{N} k_{i}\left(I-T_{i}\right)\right) y_{n-1}\right\| \\
& +\left|\gamma_{n}-\gamma_{n-1}\right|\left\|P_{C}\left(I-\lambda_{n-1}\left(\sum_{i=1}^{N} k_{i}\left(I-T_{i}\right)\right)\right) y_{n-1}\right\| \\
& \leq\left(1-\alpha_{n}\right)\left\|x_{n}-x_{n-1}\right\|+\left|\alpha_{n}-\alpha_{n-1}\right| M+\left|\beta_{n}-\beta_{n-1}\right| M+\lambda_{n} M \\
& +\left|\lambda_{n}-\lambda_{n-1}\right| M+\left|\gamma_{n}-\gamma_{n-1}\right| M,
\end{aligned}
$$

where

$$
\begin{aligned}
M:= & \max _{n \in \mathbb{N}}\left\{\|u\|,\left\|x_{n}\right\|,\left\|\left(\sum_{i=1}^{N} k_{i}\left(I-T_{i}\right)\right) y_{n+1}-\left(\sum_{i=1}^{N} k_{i}\left(I-T_{i}\right)\right) y_{n}\right\|,\right. \\
& \left.\left\|\left(\sum_{i=1}^{N} k_{i}\left(I-T_{i}\right)\right) y_{n}\right\|,\left\|P_{C}\left(I-\lambda_{n}\left(\sum_{i=1}^{N} k_{i}\left(I-T_{i}\right)\right)\right) y_{n}\right\|\right\} .
\end{aligned}
$$

From condition (i), (iii), (iv) and Lemma 2.6, we have

$$
\lim _{n \rightarrow \infty}\left\|x_{n+1}-x_{n}\right\|=0
$$

According to Eqs. (3.7), (3.9) and (3.10), we have

$$
\begin{aligned}
\left\|x_{n+1}-z\right\|^{2} \leq & \alpha_{n}\|u-z\|^{2}+\gamma_{n}\left\|P_{C}\left(I-\lambda_{n}\left(\sum_{i=1}^{N} k_{i}\left(I-T_{i}\right)\right)\right) y_{n}-z\right\|^{2} \\
& +\beta_{n}\left\|x_{n}-z\right\|^{2}-\beta_{n} \gamma_{n}\left\|x_{n}-P_{C}\left(I-\lambda_{n}\left(\sum_{i=1}^{N} k_{i}\left(I-T_{i}\right)\right)\right) y_{n}\right\|^{2} \\
\leq & \alpha_{n}\|u-z\|^{2}+\beta_{n}\left\|x_{n}-z\right\|^{2}+\gamma_{n}\left\|y_{n}-z\right\|^{2} \\
& -\beta_{n} \gamma_{n}\left\|x_{n}-P_{C}\left(I-\lambda_{n}\left(\sum_{i=1}^{N} k_{i}\left(I-T_{i}\right)\right)\right) y_{n}\right\|^{2} \\
\leq & \alpha_{n}\|u-z\|^{2}+\beta_{n}\left\|x_{n}-z\right\|^{2}+\gamma_{n}\left\|u_{n}-z\right\|^{2} \\
& \quad-\beta_{n} \gamma_{n}\left\|x_{n}-P_{C}\left(I-\lambda_{n}\left(\sum_{i=1}^{N} k_{i}\left(I-T_{i}\right)\right)\right) y_{n}\right\|^{2} \\
\leq & \alpha_{n}\|u-z\|^{2}+\left(1-\alpha_{n}\right)\left\|x_{n}-z\right\|^{2}+\gamma_{n} \gamma(L \gamma-1)\left\|\left(T_{s}^{F_{2}}\left(I-s f_{2}\right)-I\right) A x_{n}\right\|^{2} \\
& \quad-\beta_{n} \gamma_{n}\left\|x_{n}-P_{C}\left(I-\lambda_{n}\left(\sum_{i=1}^{N} k_{i}\left(I-T_{i}\right)\right)\right) y_{n}\right\|^{2}
\end{aligned}
$$

This implies that

$$
\begin{aligned}
& \gamma_{n} \gamma(1-L \gamma)\left\|\left(T_{s}^{F_{2}}\left(I-s f_{2}\right)-I\right) A x_{n}\right\|^{2} \\
& \quad \leq \alpha_{n}\|u-z\|^{2}+\left\|x_{n}-x_{n+1}\right\|\left(\left\|x_{n}-z\right\|+\left\|x_{n+1}-z\right\|\right) .
\end{aligned}
$$


By using condition (i) and (3.13), we have

$$
\lim _{n \rightarrow \infty}\left\|\left(T_{s}^{F_{2}}\left(I-s f_{2}\right)-I\right) A x_{n}\right\|=0
$$

By using the same method as (3.16), we have

$$
\lim _{n \rightarrow \infty}\left\|x_{n}-P_{C}\left(I-\lambda_{n}\left(\sum_{i=1}^{N} k_{i}\left(I-T_{i}\right)\right)\right) y_{n}\right\|=0 .
$$

Let $M_{n}=x_{n}+\gamma A^{*}\left(T_{s}^{F_{2}}\left(I-s f_{2}\right)-I\right) A x_{n}$. Applying the inequality (3.11), we have

$$
\left\|M_{n}-z\right\| \leq\left\|x_{n}-z\right\|
$$

Using the property of inverse strongly monotone operators and (3.18), we have

$$
\begin{aligned}
\left\|u_{n}-z\right\|^{2} & =\left\|T_{r}^{F_{1}}\left(I-r f_{1}\right) M_{n}-T_{r}^{F_{1}}\left(I-r f_{1}\right) z\right\|^{2} \\
& \leq\left\|\left(I-r f_{1}\right) M_{n}-\left(I-r f_{1}\right) z\right\|^{2} \\
& =\left\|M_{n}-z\right\|^{2}-2 r\left\langle M_{n}-z, f_{1} M_{n}-f_{1} z\right\rangle+r^{2}\left\|f_{1} M_{n}-f_{1} z\right\|^{2} \\
& \leq\left\|x_{n}-z\right\|^{2}+r(r-2 \rho)\left\|f_{1} M_{n}-f_{1} z\right\|^{2} .
\end{aligned}
$$

Substituting (3.19) in (3.15), we have

$$
\begin{aligned}
\left\|x_{n+1}-z\right\|^{2} \leq & \alpha_{n}\|u-z\|^{2}+\beta_{n}\left\|x_{n}-z\right\|^{2} \\
& +\gamma_{n}\left(\left\|x_{n}-z\right\|^{2}+r(r-2 \rho)\left\|f_{1} M_{n}-f_{1} z\right\|^{2}\right) \\
\leq & \alpha_{n}\|u-z\|^{2}+\left(1-\alpha_{n}\right)\left\|x_{n}-z\right\|^{2}+\gamma_{n} r(r-2 \rho)\left\|f_{1} M_{n}-f_{1} z\right\|^{2} .
\end{aligned}
$$

That is,

$$
\gamma_{n} r(2 \rho-r)\left\|f_{1} M_{n}-f_{1} z\right\|^{2} \leq \alpha_{n}\|u-z\|^{2}+\left\|x_{n}-x_{n+1}\right\|\left(\left\|x_{n}-z\right\|+\left\|x_{n+1}-z\right\|\right) .
$$

According to condition (i) and (3.13), we get

$$
\lim _{n \rightarrow \infty}\left\|f_{1} M_{n}-f_{1} z\right\|=0 .
$$

By the property of firmly nonexpansive mappings, we have

$$
\begin{aligned}
\left\|u_{n}-z\right\|^{2}= & \left\|T_{r}^{F_{1}}\left(I-r f_{1}\right) M_{n}-T_{r}^{F_{1}}\left(I-r f_{1}\right) z\right\|^{2} \\
\leq & \left\langle u_{n}-z,\left(I-r f_{1}\right) M_{n}-\left(I-r f_{1}\right) z\right\rangle \\
= & \frac{1}{2}\left(\left\|u_{n}-z\right\|^{2}+\left\|\left(I-r f_{1}\right) M_{n}-\left(I-r f_{1}\right) z\right\|^{2}\right. \\
& \left.-\left\|\left(u_{n}-z\right)-\left(\left(I-r f_{1}\right) M_{n}-\left(I-r f_{1}\right) z\right)\right\|^{2}\right) .
\end{aligned}
$$


That is,

$$
\begin{aligned}
\left\|u_{n}-z\right\|^{2} \leq & \left\|\left(I-r f_{1}\right) M_{n}-\left(I-r f_{1}\right) z\right\|^{2}-\left\|\left(u_{n}-M_{n}\right)+r\left(f_{1} M_{n}-f_{1} z\right)\right\|^{2} \\
\leq & \left\|M_{n}-z\right\|^{2}-\left(\left\|u_{n}-M_{n}\right\|^{2}+2 r\left\langle u_{n}-M_{n}, f_{1} M_{n}-f_{1} z\right\rangle\right. \\
& \left.+r^{2}\left\|f_{1} M_{n}-f_{1} z\right\|^{2}\right) \\
\leq & \left\|M_{n}-z\right\|^{2}-\left\|u_{n}-M_{n}\right\|^{2}+2 r\left\|u_{n}-M_{n}\right\|\left\|f_{1} M_{n}-f_{1} z\right\| \\
& -r^{2}\left\|f_{1} M_{n}-f_{1} z\right\|^{2} .
\end{aligned}
$$

Substituting (3.22) in (3.15), we get

$$
\begin{aligned}
\left\|x_{n+1}-z\right\|^{2} \leq & \alpha_{n}\|u-z\|^{2}+\beta_{n}\left\|x_{n}-z\right\|^{2}+\gamma_{n}\left(\left\|M_{n}-z\right\|^{2}-\left\|u_{n}-M_{n}\right\|^{2}\right. \\
& \left.+2 r\left\|u_{n}-M_{n}\right\|\left\|f_{1} M_{n}-f_{1} z\right\|-r^{2}\left\|f_{1} M_{n}-f_{1} z\right\|^{2}\right) \\
\leq & \alpha_{n}\|u-z\|^{2}+\left(1-\alpha_{n}\right)\left\|x_{n}-z\right\|^{2}-\gamma_{n}\left\|u_{n}-M_{n}\right\|^{2} \\
& +2 r \gamma_{n}\left\|u_{n}-M_{n}\right\|\left\|f_{1} M_{n}-f_{1} z\right\| .
\end{aligned}
$$

It follows that

$$
\begin{aligned}
\gamma_{n}\left\|u_{n}-M_{n}\right\|^{2} \leq & \alpha_{n}\|u-z\|^{2}+\left\|x_{n}-x_{n+1}\right\|\left(\left\|x_{n}-z\right\|+\left\|x_{n+1}-z\right\|\right) \\
& +2 r \gamma_{n}\left\|u_{n}-M_{n}\right\|\left\|f_{1} M_{n}-f_{1} z\right\| .
\end{aligned}
$$

From condition (i), (3.13) and (3.20), we ensure that

$$
\lim _{n \rightarrow \infty}\left\|u_{n}-M_{n}\right\|=0
$$

From (3.16) and (3.23), we also have

$$
\begin{aligned}
\left\|u_{n}-x_{n}\right\| & \leq\left\|u_{n}-M_{n}\right\|+\left\|M_{n}-x_{n}\right\| \\
& =\left\|u_{n}-M_{n}\right\|+\left\|x_{n}+\gamma A^{*}\left(T_{s}^{F_{2}}\left(I-s f_{2}\right)-I\right) A x_{n}-x_{n}\right\| \\
& \leq\left\|u_{n}-M_{n}\right\|+\gamma\|A\|\left\|\left(T_{s}^{F_{2}}\left(I-s f_{2}\right)-I\right) A x_{n}\right\| .
\end{aligned}
$$

Then we have

$$
\lim _{n \rightarrow \infty}\left\|u_{n}-x_{n}\right\|=0
$$

By using the same method as (3.19), we have

$$
\left\|P_{C}\left(I-d_{2} D_{2}\right) u_{n}-z\right\|^{2} \leq\left\|x_{n}-z\right\|^{2}+d_{2}\left(d_{2}-2 \beta\right)\left\|D_{2} u_{n}-D_{2} z\right\|^{2} .
$$

Substituting (3.8) and (3.25) in (3.14), we have

$$
\begin{aligned}
\left\|x_{n+1}-z\right\|^{2} \leq & \alpha_{n}\|u-z\|^{2}+\beta_{n}\left\|x_{n}-z\right\|^{2}+\gamma_{n}\left(a\left\|u_{n}-z\right\|^{2}\right. \\
& \left.+(1-a)\left\|P_{C}\left(I-d_{2} D_{2}\right) u_{n}-z\right\|^{2}\right)
\end{aligned}
$$




$$
\begin{aligned}
\leq & \alpha_{n}\|u-z\|^{2}+\left(1-\alpha_{n}\right)\left\|x_{n}-z\right\|^{2} \\
& +\gamma_{n}(1-a) d_{2}\left(d_{2}-2 \beta\right)\left\|D_{2} u_{n}-D_{2} z\right\|^{2} .
\end{aligned}
$$

We can conclude that

$$
\begin{aligned}
& \gamma_{n}(1-a) d_{2}\left(2 \beta-d_{2}\right)\left\|D_{2} u_{n}-D_{2} z\right\|^{2} \\
& \quad \leq \alpha_{n}\|u-z\|^{2}+\left\|x_{n}-x_{n+1}\right\|\left(\left\|x_{n}-z\right\|+\left\|x_{n+1}-z\right\|\right) .
\end{aligned}
$$

According to condition (i) and (3.13), we get

$$
\lim _{n \rightarrow \infty}\left\|D_{2} u_{n}-D_{2} z\right\|=0
$$

Since $P_{C}$ is a firmly nonexpansive mapping and using the same method as (3.21), we get

$$
\begin{aligned}
\left\|P_{C}\left(I-d_{2} D_{2}\right) u_{n}-z\right\|^{2} & \\
\leq & \frac{1}{2}\left(\left\|P_{C}\left(I-d_{2} D_{2}\right) u_{n}-z\right\|^{2}+\left\|\left(I-d_{2} D_{2}\right) u_{n}-\left(I-d_{2} D_{2}\right) z\right\|^{2}\right. \\
& \left.-\left\|P_{C}\left(I-d_{2} D_{2}\right) u_{n}-z-\left(I-d_{2} D_{2}\right) u_{n}+\left(I-d_{2} D_{2}\right) z\right\|^{2}\right) .
\end{aligned}
$$

That is,

$$
\begin{aligned}
\left\|P_{C}\left(I-d_{2} D_{2}\right) u_{n}-z\right\|^{2} \leq & \left\|u_{n}-z\right\|^{2}-\left\|\left(P_{C}\left(I-d_{2} D_{2}\right) u_{n}-u_{n}\right)+d_{2}\left(D_{2} u_{n}-D_{2} z\right)\right\|^{2} \\
\leq & \left\|x_{n}-z\right\|^{2}-\left\|P_{C}\left(I-d_{2} D_{2}\right) u_{n}-u_{n}\right\|^{2} \\
& +2 d_{2}\left\|P_{C}\left(I-d_{2} D_{2}\right) u_{n}-u_{n}\right\|\left\|D_{2} u_{n}-D_{2} z\right\| \\
& -d_{2}^{2}\left\|D_{2} u_{n}-D_{2} z\right\|^{2} .
\end{aligned}
$$

Substituting (3.8) and (3.27) in (3.14), we have

$$
\begin{aligned}
&\left\|x_{n+1}-z\right\|^{2} \\
& \leq \alpha_{n}\|u-z\|^{2}+\beta_{n}\left\|x_{n}-z\right\|^{2}+\gamma_{n}\left(a\left\|u_{n}-z\right\|^{2}+(1-a)\left\|P_{C}\left(I-d_{2} D_{2}\right) u_{n}-z\right\|^{2}\right) \\
& \leq \alpha_{n}\|u-z\|^{2}+\beta_{n}\left\|x_{n}-z\right\|^{2}+\gamma_{n}\left(a\left\|x_{n}-z\right\|^{2}+(1-a)\left(\left\|x_{n}-z\right\|^{2}\right.\right. \\
&-\left\|P_{C}\left(I-d_{2} D_{2}\right) u_{n}-u_{n}\right\|^{2}+2 d_{2}\left\|P_{C}\left(I-d_{2} D_{2}\right) u_{n}-u_{n}\right\|\left\|D_{2} u_{n}-D_{2} z\right\| \\
&\left.\left.-d_{2}^{2}\left\|D_{2} u_{n}-D_{2} z\right\|^{2}\right)\right) \\
& \leq \alpha_{n}\|u-z\|^{2}+\left(1-\alpha_{n}\right)\left\|x_{n}-z\right\|^{2}-\gamma_{n}(1-a)\left\|P_{C}\left(I-d_{2} D_{2}\right) u_{n}-u_{n}\right\|^{2} \\
&+2 d_{2} \gamma_{n}(1-a)\left\|P_{C}\left(I-d_{2} D_{2}\right) u_{n}-u_{n}\right\|\left\|D_{2} u_{n}-D_{2} z\right\| .
\end{aligned}
$$

Therefore

$$
\begin{aligned}
& \gamma_{n}(1-a)\left\|P_{C}\left(I-d_{2} D_{2}\right) u_{n}-u_{n}\right\|^{2} \\
& \quad \leq \alpha_{n}\|u-z\|^{2}+\left\|x_{n}-x_{n+1}\right\|\left(\left\|x_{n}-z\right\|+\left\|x_{n+1}-z\right\|\right) \\
& \quad+2 d_{2} \gamma_{n}(1-a)\left\|P_{C}\left(I-d_{2} D_{2}\right) u_{n}-u_{n}\right\|\left\|D_{2} u_{n}-D_{2} z\right\| .
\end{aligned}
$$


From condition (i), (3.13) and (3.26), we get

$$
\lim _{n \rightarrow \infty}\left\|P_{C}\left(I-d_{2} D_{2}\right) u_{n}-u_{n}\right\|=0
$$

Let $k_{n}=a u_{n}+(1-a) P_{C}\left(I-d_{2} D_{2}\right) u_{n}$. By using the same method as (3.19), we have

$$
\left\|y_{n}-z\right\|^{2} \leq\left\|x_{n}-z\right\|^{2}+d_{1}\left(d_{1}-2 \alpha\right)\left\|D_{1} k_{n}-D_{1} z\right\|^{2}
$$

Substituting (3.29) in (3.14), we have

$$
\begin{aligned}
& \left\|x_{n+1}-z\right\|^{2} \\
& \quad \leq \alpha_{n}\|u-z\|^{2}+\beta_{n}\left\|x_{n}-z\right\|^{2}+\gamma_{n}\left(\left\|x_{n}-z\right\|^{2}+d_{1}\left(d_{1}-2 \alpha\right)\left\|D_{1} k_{n}-D_{1} z\right\|^{2}\right) \\
& \quad \leq \alpha_{n}\|u-z\|^{2}+\left(1-\alpha_{n}\right)\left\|x_{n}-z\right\|^{2}+d_{1}\left(d_{1}-2 \alpha\right) \gamma_{n}\left\|D_{1} k_{n}-D_{1} z\right\|^{2} .
\end{aligned}
$$

This implies that

$$
d_{1}\left(2 \alpha-d_{1}\right) \gamma_{n}\left\|D_{1} k_{n}-D_{1} z\right\|^{2} \leq \alpha_{n}\|u-z\|^{2}+\left\|x_{n}-x_{n+1}\right\|\left(\left\|x_{n}-z\right\|+\left\|x_{n+1}-z\right\|\right) .
$$

According to condition (i) and (3.13), we have

$$
\lim _{n \rightarrow \infty}\left\|D_{1} k_{n}-D_{1} z\right\|=0
$$

By using the same method as (3.21), we have

$$
\begin{aligned}
\left\|y_{n}-z\right\|^{2} \leq & \frac{1}{2}\left(\left\|y_{n}-z\right\|^{2}+\left\|\left(I-d_{1} D_{1}\right) k_{n}-\left(I-d_{1} D_{1}\right) z\right\|^{2}\right. \\
& \left.-\left\|\left(y_{n}-k_{n}\right)+d_{1}\left(D_{1} k_{n}-D_{1} z\right)\right\|^{2}\right) .
\end{aligned}
$$

That is,

$$
\begin{aligned}
\left\|y_{n}-z\right\|^{2} \leq & \left\|k_{n}-z\right\|^{2}-\left(\left\|y_{n}-k_{n}\right\|^{2}+2 d_{1}\left\langle y_{n}-k_{n}, D_{1} k_{n}-D_{1} z\right\rangle\right. \\
& \left.+d_{1}^{2}\left\|D_{1} k_{n}-D_{1} z\right\|^{2}\right) \\
\leq & \left\|x_{n}-z\right\|^{2}-\left\|y_{n}-k_{n}\right\|^{2}+2 d_{1}\left\|y_{n}-k_{n}\right\|\left\|D_{1} k_{n}-D_{1} z\right\| \\
& -d_{1}^{2}\left\|D_{1} k_{n}-D_{1} z\right\|^{2} .
\end{aligned}
$$

Substituting (3.31) in (3.14), we have

$$
\begin{aligned}
\left\|x_{n+1}-z\right\|^{2} \leq & \alpha_{n}\|u-z\|^{2}+\beta_{n}\left\|x_{n}-z\right\|^{2}+\gamma_{n}\left(\left\|x_{n}-z\right\|^{2}-\left\|y_{n}-k_{n}\right\|^{2}\right. \\
& \left.+2 d_{1}\left\|y_{n}-k_{n}\right\|\left\|D_{1} k_{n}-d_{1} z\right\|-d_{1}^{2}\left\|D_{1} k_{n}-D_{1} z\right\|^{2}\right) \\
\leq & \alpha_{n}\|u-z\|^{2}+\left(1-\alpha_{n}\right)\left\|x_{n}-z\right\|^{2}-\gamma_{n}\left\|y_{n}-k_{n}\right\|^{2} \\
& +2 \gamma_{n} d_{1}\left\|y_{n}-k_{n}\right\|\left\|D_{1} k_{n}-D_{1} z\right\| .
\end{aligned}
$$


This implies that

$$
\begin{aligned}
\gamma_{n}\left\|y_{n}-k_{n}\right\|^{2} \leq & \alpha_{n}\|u-z\|^{2}+\left\|x_{n}-x_{n+1}\right\|\left(\left\|x_{n}-z\right\|+\left\|x_{n+1}-z\right\|\right) \\
& +2 \gamma_{n} d_{1}\left\|y_{n}-k_{n}\right\|\left\|D_{1} k_{n}-D_{1} z\right\| .
\end{aligned}
$$

According to condition (i), (3.13) and (3.30), we get

$$
\lim _{n \rightarrow \infty}\left\|y_{n}-k_{n}\right\|=0
$$

From (3.28) and (3.33)

$$
\begin{aligned}
\left\|y_{n}-u_{n}\right\| & \leq\left\|y_{n}-k_{n}\right\|+\left\|k_{n}-u_{n}\right\| \\
& \leq\left\|y_{n}-k_{n}\right\|+(1-a)\left\|P_{C}\left(I-d_{2} D_{2}\right) u_{n}-u_{n}\right\|,
\end{aligned}
$$

we conclude that

$$
\lim _{n \rightarrow \infty}\left\|y_{n}-u_{n}\right\|=0
$$

By (3.24) and (3.34), we also conclude that

$$
\lim _{n \rightarrow \infty}\left\|y_{n}-x_{n}\right\|=0
$$

Afterward, we show that $\limsup _{n \rightarrow \infty}\left\langle u-z, x_{n}-z\right\rangle \leq 0$, where $z=P_{\mathcal{F}} u$.

To show this, choose a subsequence $\left\{x_{n_{j}}\right\}$ of $\left\{x_{n}\right\}$ such that

$$
\limsup _{n \rightarrow \infty}\left\langle u-z, x_{n}-z\right\rangle=\lim _{j \rightarrow \infty}\left\langle u-z, x_{n_{j}}-z\right\rangle
$$

Without loss of generality, we may assume that $x_{n_{j}} \rightarrow \omega$ as $j \rightarrow \infty$. From (3.35), we obtain $y_{n_{j}} \rightarrow \omega$ as $j \rightarrow \infty$. From Lemma 2.3, we have $V I\left(C, D_{1}\right)=F\left(P_{C}\left(I-d_{1} D_{1}\right)\right)$. Assume that $\omega \notin V I\left(C, D_{1}\right)$, we have $\omega \neq P_{C}\left(I-d_{1} D_{1}\right) \omega$. Using Opial's condition, (3.33), we obtain

$$
\begin{aligned}
\liminf _{j \rightarrow \infty}\left\|y_{n_{j}}-\omega\right\|< & \liminf _{j \rightarrow \infty}\left\|y_{n_{j}}-P_{C}\left(I-d_{1} D_{1}\right) \omega\right\| \\
\leq & \liminf _{j \rightarrow \infty}\left(\left\|P_{C}\left(I-d_{1} D_{1}\right) k_{n_{j}}-P_{C}\left(I-d_{1} D_{1}\right) y_{n_{j}}\right\|\right. \\
& \left.+\left\|P_{C}\left(I-d_{1} D_{1}\right) y_{n_{j}}-P_{C}\left(I-d_{1} D_{1}\right) \omega\right\|\right) \\
\leq & \liminf _{j \rightarrow \infty}\left(\left\|k_{n_{j}}-y_{n_{j}}\right\|+\left\|y_{n_{j}}-\omega\right\|\right) \\
\leq & \liminf _{j \rightarrow \infty}\left\|y_{n_{j}}-\omega\right\| .
\end{aligned}
$$

This is a contradiction, so we have 
From (3.24), we have $u_{n_{j}} \rightarrow \omega$ as $j \rightarrow \infty$. By (3.28) and using the same method as (3.37), we obtain

$$
\omega \in \operatorname{VI}\left(C, D_{2}\right)
$$

Next, we show that $\omega \in \bigcap_{i=1}^{N} F\left(T_{i}\right)$. From Lemma 2.5, we have

$$
\bigcap_{i=1}^{N} F\left(T_{i}\right)=F\left(P_{C}\left(I-\lambda_{n_{j}}\left(\sum_{i=1}^{N} k_{i}\left(I-T_{i}\right)\right)\right)\right) .
$$

Assume that $\omega \notin \bigcap_{i=1}^{N} F\left(T_{i}\right)$, and that $\omega \neq P_{C}\left(I-\lambda_{n_{j}}\left(\sum_{i=1}^{N} k_{i}\left(I-T_{i}\right)\right)\right) \omega$. Using Opial's condition, (3.17) and (3.35), we obtain

$$
\begin{aligned}
& \liminf _{j \rightarrow \infty}\left\|x_{n_{j}}-\omega\right\| \\
& <\liminf _{j \rightarrow \infty} \| x_{n_{j}}-P_{C}\left(I-\lambda_{n_{j}}\left(\sum_{i=1}^{N} k_{i}\left(I-T_{i}\right)\right) \omega \|\right. \\
& \leq \liminf _{j \rightarrow \infty}\left(\left\|x_{n_{j}}-P_{C}\left(I-\lambda_{n_{j}}\left(\sum_{i=1}^{N} k_{i}\left(I-T_{i}\right)\right)\right) y_{n_{j}}\right\|\right. \\
& +\left\|P_{C}\left(I-\lambda_{n_{j}}\left(\sum_{i=1}^{N} k_{i}\left(I-T_{i}\right)\right)\right) y_{n_{j}}-P_{C}\left(I-\lambda_{n_{j}}\left(\sum_{i=1}^{N} k_{i}\left(I-T_{i}\right)\right)\right) x_{n_{j}}\right\| \\
& +\| P_{C}\left(I-\lambda_{n_{j}}\left(\sum_{i=1}^{N} k_{i}\left(I-T_{i}\right)\right)\right) x_{n_{j}}-P_{C}\left(I-\lambda_{n_{j}}\left(\sum_{i=1}^{N} k_{i}\left(I-T_{i}\right)\right) \omega \|\right) \\
& \leq \liminf _{j \rightarrow \infty}\left(\left\|y_{n_{j}}-x_{n_{j}}\right\|+\lambda_{n_{j}}\left\|\left(\sum_{i=1}^{N} k_{i}\left(I-T_{i}\right)\right) y_{n_{j}}-\left(\sum_{i=1}^{N} k_{i}\left(I-T_{i}\right)\right) x_{n_{j}}\right\|\right. \\
& \left.+\left\|x_{n_{j}}-\omega\right\|+\lambda_{n_{j}}\left\|\left(\sum_{i=1}^{N} k_{i}\left(I-T_{i}\right)\right) x_{n_{j}}-\left(\sum_{i=1}^{N} k_{i}\left(I-T_{i}\right)\right) \omega\right\|\right) \\
& \leq \liminf _{j \rightarrow \infty}\left\|x_{n_{j}}-\omega\right\| .
\end{aligned}
$$

This is a contradiction, so we have

$$
\omega \in \bigcap_{i=1}^{N} F\left(T_{i}\right)
$$

After that, we show that $\omega \in \Omega$. Assume $\omega \notin E P\left(F_{1}, f_{1}\right)$. Since $E P\left(F_{1}, f_{1}\right)=F\left(T_{r}^{F_{1}}\left(I-r f_{1}\right)\right)$, we obtain $\omega \neq T_{r}^{F_{1}}\left(I-r f_{1}\right) \omega$. Using Opial's condition and (3.23), we get

$$
\begin{aligned}
\liminf _{j \rightarrow \infty}\left\|u_{n_{j}}-\omega\right\|< & \liminf _{j \rightarrow \infty}\left\|u_{n_{j}}-T_{r}^{F_{1}}\left(I-r f_{1}\right) \omega\right\| \\
\leq & \liminf _{j \rightarrow \infty}\left(\left\|T_{r}^{F_{1}}\left(I-r f_{1}\right) M_{n_{j}}-T_{r}^{F_{1}}\left(I-r f_{1}\right) u_{n_{j}}\right\|\right. \\
& \left.+\left\|T_{r}^{F_{1}}\left(I-r f_{1}\right) u_{n_{j}}-T_{r}^{F_{1}}\left(I-r f_{1}\right) \omega\right\|\right)
\end{aligned}
$$




$$
\begin{aligned}
& \leq \liminf _{j \rightarrow \infty}\left(\left\|M_{n_{j}}-u_{n_{j}}\right\|+\left\|u_{n_{j}}-\omega\right\|\right) \\
& \leq \liminf _{j \rightarrow \infty}\left\|u_{n_{j}}-\omega\right\| .
\end{aligned}
$$

This is a contradiction, so we have

$$
\omega \in \operatorname{EP}\left(F_{1}, f_{1}\right)
$$

Next, we show that $A \omega \in E P\left(F_{2}, f_{2}\right)$. Since $A$ is bounded linear operator so that $A x_{n_{j}} \rightarrow$ $A \omega$ as $j \rightarrow \infty$. Assume $A \omega \notin E P\left(F_{2}, f_{2}\right)$. Since $E P\left(F_{2}, f_{2}\right)=F\left(T_{s}^{F_{2}}\left(I-s f_{2}\right)\right)$, we obtain $A \omega \neq$ $T_{s}^{F_{2}}\left(I-s f_{s}\right) A \omega$. Using Opial's condition and (3.16), we have

$$
A \omega \in E P\left(F_{2}, f_{2}\right) .
$$

We can conclude that $\omega \in \Omega$. Therefore $\omega \in \mathcal{F}$. Since $x_{n_{j}} \rightarrow \omega$ as $j \rightarrow \infty$, we have

$$
\begin{aligned}
\limsup _{n \rightarrow \infty}\left\langle u-z, x_{n}-z\right\rangle & =\lim _{j \rightarrow \infty}\left\langle u-z, x_{n_{j}}-z\right\rangle \\
& =\langle u-z, \omega-z\rangle \leq 0 .
\end{aligned}
$$

Finally, we show that the sequence $\left\{x_{n}\right\}$ converges strongly to $z=P_{\mathcal{F}} u$. By (3.7), (3.9) and (3.11), we get

$$
\begin{aligned}
\left\|x_{n+1}-z\right\|^{2}= & \left\|\alpha_{n}(u-z)+\beta_{n}\left(x_{n}-z\right)+\gamma_{n}\left(P_{C}\left(I-\lambda_{n}\left(\sum_{i=1}^{N} k_{i}\left(I-T_{i}\right)\right)\right) y_{n}-z\right)\right\|^{2} \\
\leq & \left\|\beta_{n}\left(x_{n}-z\right)+\gamma_{n}\left(P_{C}\left(I-\lambda_{n}\left(\sum_{i=1}^{N} k_{i}\left(I-T_{i}\right)\right)\right) y_{n}-z\right)\right\|^{2} \\
& +2 \alpha_{n}\left\langle u-z, x_{n+1}-z\right\rangle \\
\leq & \left(\beta_{n}\left\|x_{n}-z\right\|+\gamma_{n}\left\|u_{n}-z\right\|\right)^{2}+2 \alpha_{n}\left\langle u-z, x_{n+1}-z\right\rangle \\
\leq & \left(1-\alpha_{n}\right)\left\|x_{n}-z\right\|^{2}+2 \alpha_{n}\left\langle u-z, x_{n+1}-z\right\rangle .
\end{aligned}
$$

According to condition (i), (3.42) and Lemma 2.6, we can conclude that $\left\{x_{n}\right\}$ converges strongly to $z=P_{\mathcal{F}} u$. By (3.24) and (3.35), we have $\left\{u_{n}\right\}$ and $\left\{y_{n}\right\}$ converge strongly to $z=$ $P_{\mathcal{F}} u$. This completes the proof.

These results are directly proved from Theorem 3.4. Therefore, we omit the proof.

Corollary 3.5 Let $C$ and $Q$ be nonempty closed convex subsets of a real Hilbert space $H_{1}$ and $H_{2}$, respectively. Let $A: H_{1} \rightarrow H_{2}$ be a bounded linear operator. Let $D_{1}, D_{2}: C \rightarrow H_{1}$ be $\alpha, \beta$-inverse strongly monotone mappings, respectively. Let $F_{1}: C \times C \rightarrow \mathbb{R}$ and $F_{2}: Q \times$ $Q \rightarrow \mathbb{R}$ be the bifunctions satisfying (A1)-(A4). Let $T$ be a quasi-nonexpansive mapping of $C$ into itself. Let $f_{1}: H_{1} \rightarrow H_{1}$ be a $\rho$-inverse strongly monotone mapping and $f_{2}: H_{2} \rightarrow H_{2}$ be a firmly nonexpansive mapping. Assume $\mathcal{F}=V I\left(C, D_{1}\right) \cap V I\left(C, D_{2}\right) \cap F(T) \cap \Omega \neq \emptyset$. For 
given $x_{1}, u \in C$, and let $\left\{x_{n}\right\},\left\{u_{n}\right\}$ and $\left\{y_{n}\right\}$ be sequences generated by

$$
\left\{\begin{array}{l}
u_{n}=T_{r}^{F_{1}}\left(I-r f_{1}\right)\left(x_{n}+\gamma A^{*}\left(T_{s}^{F_{2}}\left(I-s f_{2}\right)-I\right) A x_{n}\right), \\
y_{n}=P_{C}\left(I-d_{1} D_{1}\right)\left(a u_{n}+(1-a) P_{C}\left(I-d_{2} D_{2}\right) u_{n}\right), \\
x_{n+1}=\alpha_{n} u+\beta_{n} x_{n}+\gamma_{n} P_{C}\left(I-\lambda_{n}(I-T)\right) y_{n}, \quad \forall n \in \mathbb{N},
\end{array}\right.
$$

where $d_{1} \in(0,2 \alpha), d_{2} \in(0,2 \beta), r \in(0,2 \rho), s \in(0,1), a \in[0,1], \gamma \in(0,1 / L), L$ is the spectral radius of the operator $A^{*} A$ and $A^{*}$ is the adjoint of $A$. Also $\left\{\alpha_{n}\right\},\left\{\beta_{n}\right\},\left\{\gamma_{n}\right\}$ are sequences in $[0,1]$ with $\alpha_{n}+\beta_{n}+\gamma_{n}=1$ for all $n \in \mathbb{N}$. Suppose the conditions (i)-(iv) of Theorem 3.4 hold. Then $\left\{x_{n}\right\},\left\{u_{n}\right\}$ and $\left\{y_{n}\right\}$ converge strongly to $z=P_{\mathcal{F}} u$.

Corollary 3.6 Let $C$ be nonempty closed convex subset of a real Hilbert space $H_{1}$. Let $D_{1}, D_{2}: C \rightarrow H_{1}$ be $\alpha, \beta$-inverse strongly monotone mappings, respectively. Let $F_{1}: C \times C \rightarrow$ $\mathbb{R}$ be the bifunction satisfying (A1)-(A4). Let $\left\{T_{i}\right\}_{i=1}^{N}$ be a finite family of quasi-nonexpansive mappings of $C$ into itself with $\bigcap_{i=1}^{N} F\left(T_{i}\right) \neq \emptyset$. Let $f_{1}: H_{1} \rightarrow H_{1}$ be a $\rho$-inverse strongly monotone mapping. Assume $\mathcal{F}=V I\left(C, D_{1}\right) \cap V I\left(C, D_{2}\right) \cap \bigcap_{i=1}^{N} F\left(T_{i}\right) \cap E P\left(F_{1}, f_{1}\right) \neq \emptyset$. For given $x_{1}, u \in C$ and let $\left\{x_{n}\right\},\left\{u_{n}\right\}$ and $\left\{y_{n}\right\}$ be sequences generated by

$$
\left\{\begin{array}{l}
u_{n}=T_{r}^{F_{1}}\left(I-r f_{1}\right) x_{n}, \\
y_{n}=P_{C}\left(I-d_{1} D_{1}\right)\left(a u_{n}+(1-a) P_{C}\left(I-d_{2} D_{2}\right) u_{n}\right), \\
x_{n+1}=\alpha_{n} u+\beta_{n} x_{n}+\gamma_{n} P_{C}\left(I-\lambda_{n}\left(\sum_{i=1}^{N} k_{i}\left(I-T_{i}\right)\right)\right) y_{n}, \quad \forall n \in \mathbb{N},
\end{array}\right.
$$

where $d_{1} \in(0,2 \alpha), d_{2} \in(0,2 \beta), r \in(0,2 \rho), a \in[0,1], 0<k_{i}<1$ with $\sum_{i=1}^{N} k_{i}=1$. Also $\left\{\alpha_{n}\right\}$, $\left\{\beta_{n}\right\},\left\{\gamma_{n}\right\}$ are sequences in $[0,1]$ with $\alpha_{n}+\beta_{n}+\gamma_{n}=1$ for all $n \in \mathbb{N}$. Suppose the conditions (i)-(iv) of Theorem 3.4 hold. Then $\left\{x_{n}\right\},\left\{u_{n}\right\}$ and $\left\{y_{n}\right\}$ converge strongly to $z=P_{\mathcal{F}} u$.

Corollary 3.7 Let $C$ and $Q$ be nonempty closed convex subsets of a real Hilbert space $H_{1}$ and $H_{2}$, respectively. Let $A: H_{1} \rightarrow H_{2}$ be a bounded linear operator. Let $D_{1}, D_{2}: C \rightarrow$ $H_{1}$ be $\alpha, \beta$-inverse strongly monotone mappings, respectively. Let $F_{1}: C \times C \rightarrow \mathbb{R}$ and $F_{2}: Q \times Q \rightarrow \mathbb{R}$ be the bifunctions satisfying (A1)-(A4). Let $\left\{T_{i}\right\}_{i=1}^{N}$ be a finite family of quasi-nonexpansive mappings of $C$ into itself with $\bigcap_{i=1}^{N} F\left(T_{i}\right) \neq \emptyset$. Assume $\mathcal{F}=V I\left(C, D_{1}\right) \cap$ $V I\left(C, D_{2}\right) \cap \bigcap_{i=1}^{N} F\left(T_{i}\right) \cap \Gamma \neq \emptyset$. For given $x_{1}, u \in C$ and let $\left\{x_{n}\right\},\left\{u_{n}\right\}$ and $\left\{y_{n}\right\}$ be sequences generated by

$$
\left\{\begin{array}{l}
u_{n}=T_{r}^{F_{1}}\left(x_{n}+\gamma A^{*}\left(T_{s}^{F_{2}}-I\right) A x_{n}\right), \\
y_{n}=P_{C}\left(I-d_{1} D_{1}\right)\left(a u_{n}+(1-a) P_{C}\left(I-d_{2} D_{2}\right) u_{n}\right), \\
x_{n+1}=\alpha_{n} u+\beta_{n} x_{n}+\gamma_{n} P_{C}\left(I-\lambda_{n}\left(\sum_{i=1}^{N} k_{i}\left(I-T_{i}\right)\right)\right) y_{n}, \quad \forall n \in \mathbb{N},
\end{array}\right.
$$

where $d_{1} \in(0,2 \alpha), d_{2} \in(0,2 \beta), a \in[0,1], 0<k_{i}<1$ with $\sum_{i=1}^{N} k_{i}=1, \gamma \in(0,1 / L), L$ is the spectral radius of the operator $A^{*} A$ and $A^{*}$ is the adjoint of $A$. Also $\left\{\alpha_{n}\right\},\left\{\beta_{n}\right\},\left\{\gamma_{n}\right\}$ are sequences in $[0,1]$ with $\alpha_{n}+\beta_{n}+\gamma_{n}=1$ for all $n \in \mathbb{N}$. Suppose the conditions (i)-(iv) of Theorem 3.4 hold. Then $\left\{x_{n}\right\},\left\{u_{n}\right\}$ and $\left\{y_{n}\right\}$ converge strongly to $z=P_{\mathcal{F}} u$.

Remark 3.8 If we take $N=1$ in Theorem 3.4, we have a strong convergence for finding a common element of the set of solutions of variational inequality problems and the set 
of fixed points of a quasi-nonexpansive mapping and the set of solutions of the modified split generalized equilibrium problem. From previous result, we can apply by using the same method as Theorem 4.5 in [24]. We have a strong convergence for finding a common element of the set of solutions of variational inequality problems and the set of fixed points of a finite family of nonspreading mappings and the set of solutions of the modified split generalized equilibrium problem. By using our main result, Theorem 3.4 reduces to the Corollary 3.6, the solution of the generalized equilibrium problem and Corollary 3.7, the split equilibrium problem. All theorems are found as regards the solution of common fixed points of a finite family of quasi-nonexpansive mappings without assuming $T_{\omega}:=(1-\omega) I+$ $\omega T$ and $T$ is demiclosed; a difficult proof in a framework of Hilbert space.

\section{Application}

The following knowledge is used to prove Theorem 4.4. A mapping $T: C \rightarrow C$ is called nonspreading if

$$
2\|T x-T y\|^{2} \leq\|T x-y\|^{2}+\|T y-x\|^{2}, \quad \forall x, y \in C .
$$

Such a mapping is defined by Kohsaka and Takahashi [25].

In 2009, Iemoto and Takahashi [26] proved that (4.1) is equivalent to

$$
\|T x-T y\|^{2} \leq\|x-y\|^{2}+2\langle x-T x, y-T y\rangle, \quad \forall x, y \in C .
$$

Remark 4.1 A nonspreading mapping $T$ with $F(T) \neq \emptyset$ is quasi-nonexpansive mapping $T$.

Lemma 4.2 ([25]) Let $H$ be a Hilbert space, let $C$ be a nonempty closed convex subset of $H$, and let $S$ be a nonspreading mapping of $C$ into itself. Then $F(S)$ is closed and convex.

In 2009, Kangtunyakarn and Suantai[27] introduced the $S$-mapping generated by $T_{1}, T_{2}, T_{3}, \ldots, T_{N}$ and $\lambda_{1}, \lambda_{2}, \ldots, \lambda_{N}$ as follows.

Definition 4.1 Let $C$ be a nonempty convex subset of a real Banach space. Let $\left\{T_{i}\right\}_{i=1}^{N}$ be a finite family of (nonexpansive) mappings of $C$ into itself. For each $j=1,2, \ldots, N$, let $\alpha_{j}=$ $\left(\alpha_{1}^{j}, \alpha_{2}^{j}, \alpha_{3}^{j}\right) \in I \times I \times I$, where $I \in[0,1]$ and $\alpha_{1}^{j}+\alpha_{2}^{j}+\alpha_{3}^{j}=1$. Define the mapping $S: C \rightarrow C$ as follows:

$$
\begin{aligned}
& U_{0}=I, \\
& U_{1}=\alpha_{1}^{1} T_{1} U_{0}+\alpha_{2}^{1} U_{0}+\alpha_{3}^{1} I, \\
& U_{2}=\alpha_{1}^{2} T_{2} U_{1}+\alpha_{2}^{2} U_{1}+\alpha_{3}^{2} I, \\
& U_{3}=\alpha_{1}^{3} T_{3} U_{2}+\alpha_{2}^{3} U_{2}+\alpha_{3}^{3} I, \\
& \vdots \\
& U_{N-1}=\alpha_{1}^{N-1} T_{N-1} U_{N-2}+\alpha_{2}^{N-1} U_{N-2}+\alpha_{3}^{N-1} I, \\
& S=U_{N}=\alpha_{1}^{N} T_{N} U_{N-1}+\alpha_{2}^{N} U_{N-1}+\alpha_{3}^{N} I .
\end{aligned}
$$

This mapping is called an $S$-mapping generated by $T_{1}, T_{2}, \ldots, T_{N}$ and $\alpha_{1}, \alpha_{2}, \ldots, \alpha_{N}$. 
Lemma 4.3 ([28]) Let $C$ be a nonempty closed convex subset of a real Hilbert space. Let $\left\{T_{i}\right\}_{i=1}^{N}$ be a finite family of nonspreading mappings of $C$ into $C$ with $\bigcap_{i=1}^{N} F\left(T_{i}\right) \neq \emptyset$, and let $\alpha_{j}=\left(\alpha_{1}^{j}, \alpha_{2}^{j}, \alpha_{3}^{j}\right) \in I \times I \times I, j=1,2, \ldots, N$, where $I=[0,1], \alpha_{1}^{j}+\alpha_{2}^{j}+\alpha_{3}^{j}=1, \alpha_{1}^{j}, \alpha_{3}^{j} \in(0,1)$ for all $j=1,2, \ldots, N-1$ and $\alpha_{1}^{N} \in(0,1], \alpha_{3}^{N} \in[0,1), \alpha_{2}^{j} \in[0,1)$ for all $j=1,2, \ldots, N$. Let $S$ be the mapping generated by $T_{1}, T_{2}, \ldots, T_{N}$ and $\alpha_{1}, \alpha_{2}, \ldots, \alpha_{N}$. Then $F(S)=\bigcap_{i=1}^{N} F\left(T_{i}\right)$ and $S$ is a quasi-nonexpansive mapping.

By using these results, we obtain the following theorems.

Theorem 4.4 Let $C$ and $Q$ be nonempty closed convex subsets of a real Hilbert space $H_{1}$ and $H_{2}$, respectively. Let $A: H_{1} \rightarrow H_{2}$ be a bounded linear operator. Let $D_{1}, D_{2}: C \rightarrow H_{1}$ be $\alpha, \beta$-inverse strongly monotone mappings, respectively. Let $F_{1}: C \times C \rightarrow \mathbb{R}$ and $F_{2}: Q \times$ $Q \rightarrow \mathbb{R}$ be the bifunctions satisfying (A1)-(A4). Let $\left\{T_{i}\right\}_{i=1}^{N}$ be a finite family of nonspreading mappings of $C$ into $C$ with $\bigcap_{i=1}^{N} F\left(T_{i}\right) \neq \emptyset$, and let $\alpha_{j}=\left(\alpha_{1}^{j}, \alpha_{2}^{j}, \alpha_{3}^{j}\right) \in I \times I \times I, j=1,2, \ldots, N$, where $I=[0,1], \alpha_{1}^{j}+\alpha_{2}^{j}+\alpha_{3}^{j}=1, \alpha_{1}^{j}, \alpha_{3}^{j} \in(0,1)$ for all $j=1,2, \ldots, N-1$ and $\alpha_{1}^{N} \in(0,1], \alpha_{3}^{N} \in$ $[0,1), \alpha_{2}^{j} \in[0,1)$ for all $j=1,2, \ldots, N$. Let $S$ be the mapping generated by $T_{1}, T_{2}, \ldots, T_{N}$ and $\alpha_{1}, \alpha_{2}, \ldots, \alpha_{N}$. Let $f_{1}: H_{1} \rightarrow H_{1}$ be a $\rho$-inverse strongly monotone mapping and $f_{2}: H_{2} \rightarrow H_{2}$ be a firmly nonexpansive mapping. Assume $\mathcal{F}=V I\left(C, D_{1}\right) \cap V I\left(C, D_{2}\right) \cap \bigcap_{i=1}^{N} F\left(T_{i}\right) \cap \Omega \neq \emptyset$. For given $x_{1}, u \in C$ and let $\left\{x_{n}\right\},\left\{u_{n}\right\}$ and $\left\{y_{n}\right\}$ be sequences generated by

$$
\left\{\begin{array}{l}
u_{n}=T_{r}^{F_{1}}\left(I-r f_{1}\right)\left(x_{n}+\gamma A^{*}\left(T_{s}^{F_{2}}\left(I-s f_{2}\right)-I\right) A x_{n}\right), \\
y_{n}=P_{C}\left(I-d_{1} D_{1}\right)\left(a u_{n}+(1-a) P_{C}\left(I-d_{2} D_{2}\right) u_{n}\right), \\
x_{n+1}=\alpha_{n} u+\beta_{n} x_{n}+\gamma_{n} P_{C}\left(I-\lambda_{n}(I-S)\right) y_{n}, \quad \forall n \in \mathbb{N},
\end{array}\right.
$$

where $d_{1} \in(0,2 \alpha), d_{2} \in(0,2 \beta), r \in(0,2 \rho), s \in(0,1), a \in[0,1], \gamma \in(0,1 / L), L$ is the spectral radius of the operator $A^{*} A$ and $A^{*}$ is the adjoint of $A$. Also $\left\{\alpha_{n}\right\},\left\{\beta_{n}\right\},\left\{\gamma_{n}\right\}$ are sequences in $[0,1]$ with $\alpha_{n}+\beta_{n}+\gamma_{n}=1$ for all $n \in \mathbb{N}$. Suppose the conditions (i)-(iv) of Theorem 3.4 hold. Then $\left\{x_{n}\right\},\left\{u_{n}\right\}$ and $\left\{y_{n}\right\}$ converge strongly to $z=P_{\mathcal{F}} u$.

Proof By using Corollary 3.5 and Lemma 4.3, we obtain the conclusion.

\section{Example and numerical results}

In this section, an example is given for supporting Theorem 3.4. In Example 5.1, we only instance an example in infinite dimensional Hilbert space for supporting Theorem 3.4. We omit the computer programming.

Example 5.1 Let $H_{1}=H_{2}=C=Q=\ell_{2}$ be the linear space whose elements consist of all 2-summable sequences $\left(x_{1}, x_{2}, \ldots, x_{j}, \ldots\right)$ of scalars, i.e.,

$$
\ell_{2}=\left\{x: x=\left(x_{1}, x_{2}, \ldots, x_{j}, \ldots\right) \text { and } \sum_{j=1}^{\infty}\left|x_{j}\right|^{2}<\infty\right\} \text {, }
$$

with an inner product $\langle\cdot, \cdot \cdot\rangle: \ell_{2} \times \ell_{2} \rightarrow \mathbb{R}$ defined by $\langle x, y\rangle=\sum_{j=1}^{\infty} x_{j} y_{j}$ where $x=\left\{x_{j}\right\}_{j=1}^{\infty}, y=$ $\left\{y_{j}\right\}_{j=1}^{\infty} \in \ell_{2}$ and a norm $\|\cdot\|: \ell_{2} \rightarrow \mathbb{R}$ defined by $\|x\|_{2}=\left(\sum_{j=1}^{\infty}\left|x_{j}\right|^{2}\right)^{\frac{1}{2}}$ where $x=\left\{x_{j}\right\}_{j=1}^{\infty} \in \ell_{2}$. Let the mapping $A: \ell_{2} \rightarrow \ell_{2}$ be defined by $A x=\left(\frac{x_{1}}{3}, \frac{x_{2}}{3}, \ldots, \frac{x_{j}}{3}, \ldots\right)$ for all $x=\left\{x_{j}\right\}_{j=1}^{\infty} \in \ell_{2}$ and $A^{*}: \ell_{2} \rightarrow \ell_{2}$ be defined by $A^{*} z=\left(\frac{z_{1}}{3}, \frac{z_{2}}{3}, \ldots, \frac{z_{j}}{3}, \ldots\right)$ for all $z=\left\{z_{j}\right\}_{j=1}^{\infty} \in \ell_{2}$. Let $D_{1}, D_{2}$ : 
$\ell_{2} \rightarrow \ell_{2}$ be defined by $D_{1} x=\left(\frac{x_{1}}{6}, \frac{x_{2}}{6}, \ldots, \frac{x_{j}}{6}, \ldots\right)$ and $D_{2} x=\left(\frac{x_{1}}{5}, \frac{x_{2}}{5}, \ldots, \frac{x_{j}}{5}, \ldots\right), \forall x=\left\{x_{j}\right\}_{j=1}^{\infty} \in$ $\ell_{2}$, respectively. Let the mapping $T_{i}: \ell_{2} \rightarrow \ell_{2}$ be defined by $T_{i} x=\left(\frac{3 i x_{1}}{5 i+1}, \frac{3 i x_{2}}{5 i+1}, \ldots, \frac{3 i x_{j}}{5 i+1}, \ldots\right)$, $\forall x=\left\{x_{j}\right\}_{j=1}^{\infty} \in \ell_{2}$ and $k_{i}=\frac{6}{7^{i}}+\frac{1}{N 7^{N}}$ for every $i=1,2, \ldots, N$. Let the mapping $F_{1}, F_{2}: \mathbb{R}^{2} \times$ $\mathbb{R}^{2} \rightarrow \mathbb{R}$ be defined by

$$
F_{1}(x, y)=-x^{2}+y^{2}, \quad \forall x=\left\{x_{j}\right\}_{j=1}^{\infty}, y=\left\{y_{j}\right\}_{j=1}^{\infty} \in \ell_{2},
$$

and

$$
F_{2}(x, y)=-2 x^{2}+x y+y^{2}, \quad \forall x=\left\{x_{j}\right\}_{j=1}^{\infty}, y=\left\{y_{j}\right\}_{j=1}^{\infty} \in \ell_{2} .
$$

Let the mapping $f_{1}: \ell_{2} \rightarrow \ell_{2}$ be defined by $f_{1} x=\left(\frac{x_{1}}{5}, \frac{x_{2}}{5}, \ldots, \frac{x_{j}}{5}, \ldots\right), \forall x=\left\{x_{j}\right\}_{j=1}^{\infty} \in \ell_{2}$ and the mapping $f_{2}: \ell_{2} \rightarrow \ell_{2}$ be defined by $f_{2} x=\left(\frac{x_{1}}{7}, \frac{x_{2}}{7}, \ldots, \frac{x_{j}}{7}, \ldots\right), \forall x=\left\{x_{j}\right\}_{j=1}^{\infty} \in \ell_{2}$. Let $r=1$ and $s=0.5$. Since $L=\frac{1}{9}$, we choose $\gamma=0.5$. Let $x_{1}=\left(x_{1}^{1}, x_{1}^{2}, \ldots, x_{1}^{j}, \ldots\right)$ and $u=\left(u_{1}, u_{2}, \ldots\right.$, $\left.u_{j}, \ldots\right) \in \ell_{2}$ and let the sequences $\left\{x_{n}\right\},\left\{y_{n}\right\}$ and $\left\{u_{n}\right\}$ be generated by (3.6) as follows:

$$
\left\{\begin{array}{l}
u_{n}=T_{1}^{F_{1}}\left(I-f_{1}\right)\left(x_{n}+0.5 A^{*}\left(T_{0.5}^{F_{2}}\left(I-0.5 f_{2}\right)-I\right) A x_{n}\right) \\
y_{n}=\left(I-D_{1}\right)\left(0.5 u_{n}+0.5\left(I-D_{2}\right) u_{n}\right) \\
x_{n+1}=\frac{1}{2 n} u+\frac{7 n-4}{12 n} x_{n}+\frac{5 n-2}{12 n}\left(y_{n}-\left(\left(\frac{1}{2 n^{2}}\right)\left(\sum_{i=1}^{N}\left(\frac{6}{7^{i}}+\frac{1}{N 7^{N}}\right)\left(y_{n}-T_{i} y_{n}\right)\right)\right)\right)
\end{array}\right.
$$

for all $n \geq 1$, where $x_{n}=\left(x_{n}^{1}, x_{n}^{2}, \ldots, x_{n}^{j}, \ldots\right), y_{n}=\left(y_{n}^{1}, y_{n}^{2}, \ldots, y_{n}^{j}, \ldots\right)$ and $u_{n}=\left(u_{n}^{1}, u_{n}^{2}, \ldots\right.$, $\left.u_{n}^{j}, \ldots\right)$. It easy to see that $D_{1}, D_{2}, T_{i}, F_{1}, F_{2}, f_{1}$ and $f_{2}$ satisfy Theorem 3.4. Moreover, we have $V I\left(C, D_{1}\right) \cap V I\left(C, D_{2}\right) \cap \bigcap_{i=1}^{N} F\left(T_{i}\right) \cap \Omega=\{0\}$, where $\rho=d_{1}=d_{2}=1$. From Theorem 3.4, we can conclude that the sequences $\left\{x_{n}\right\},\left\{y_{n}\right\}$ and $\left\{u_{n}\right\}$ converge strongly to 0 .

In Example 5.2, we give computer programming to support our main result.

Example 5.2 Let $H_{1}=H_{2}=C=Q=\mathbb{R}^{2}$ be the two-dimensional Euclidean space of the real number with an inner product $\langle\cdot, \cdot\rangle: \mathbb{R}^{2} \times \mathbb{R}^{2} \rightarrow \mathbb{R}$ be defined by $\langle x, y\rangle=x \cdot y=x_{1} y_{1}+x_{2} y_{2}$ where $x=\left(x_{1}, x_{2}\right) \in \mathbb{R}^{2}$ and $y=\left(y_{1}, y_{2}\right) \in \mathbb{R}^{2}$ and a usual norm $\|\cdot\|: \mathbb{R}^{2} \rightarrow \mathbb{R}$ be defined by $\|x\|=\sqrt{x_{1}^{2}+x_{2}^{2}}$ where $x=\left(x_{1}, x_{2}\right) \in \mathbb{R}^{2}$. Let the mapping $A: \mathbb{R}^{2} \rightarrow \mathbb{R}^{2}$ be defined by $A x=\left(2 x_{1}-x_{2}, x_{1}+2 x_{2}\right)$ for all $x=\left(x_{1}, x_{2}\right) \in \mathbb{R}^{2}$ and $A^{*}: \mathbb{R}^{2} \rightarrow \mathbb{R}^{2}$ be defined by $A^{*} z=\left(2 z_{1}-\right.$ $\left.z_{2}, 2 z_{2}-z_{1}\right)$ for all $z=\left(z_{1}, z_{2}\right) \in \mathbb{R}^{2}$. Let $D_{1}, D_{2}: \mathbb{R}^{2} \rightarrow \mathbb{R}^{2}$ be defined by $D_{1} x=\left(\frac{x_{1}}{6}, \frac{x_{2}}{6}\right)$ and $D_{2} x=\left(\frac{x_{1}}{2}, \frac{x_{2}}{3}\right), \forall x=\left(x_{1}, x_{2}\right) \in \mathbb{R}^{2}$, respectively. Let the mapping $T_{i}: \mathbb{R}^{2} \rightarrow \mathbb{R}^{2}$ be defined by $T_{i} x=\left(\frac{3 i x_{1}}{3 i+1}, \frac{3 i x_{2}}{3 i+2}\right), \forall x=\left(x_{1}, x_{2}\right) \in \mathbb{R}^{2}$ and $k_{i}=\frac{6}{7^{i}}+\frac{1}{N 7^{N}}$ for every $i=1,2, \ldots, N$. Let the mapping $F_{1}, F_{2}: \mathbb{R}^{2} \times \mathbb{R}^{2} \rightarrow \mathbb{R}$ be defined by

$$
F_{1}(x, y)=-x^{2}+y^{2}, \quad \forall x=\left(x_{1}, x_{2}\right), y=\left(y_{1}, y_{2}\right) \in \mathbb{R}^{2},
$$

and

$$
F_{2}(x, y)=-2 x^{2}+x y+y^{2}, \quad \forall x=\left(x_{1}, x_{2}\right), y=\left(y_{1}, y_{2}\right) \in \mathbb{R}^{2} .
$$

Let the mapping $f_{1}: \mathbb{R}^{2} \rightarrow \mathbb{R}^{2}$ be defined by $f_{1} x=\left(\frac{x_{1}}{5}, \frac{x_{2}}{5}\right), \forall x=\left(x_{1}, x_{2}\right) \in \mathbb{R}^{2}$ and the mapping $f_{2}: \mathbb{R}^{2} \rightarrow \mathbb{R}^{2}$ be defined by $f_{2} x=\left(\frac{x_{1}}{7}, \frac{x_{2}}{7}\right), \forall x=\left(x_{1}, x_{2}\right) \in \mathbb{R}^{2}$. Let $r=1$ and $s=0.5$, the 
sequences $z_{n}=\left(z_{n}^{1}, z_{n}^{2}\right), x_{n}=\left(x_{n}^{1}, x_{n}^{2}\right), u_{n}=\left(u_{n}^{1}, u_{n}^{2}\right), y=\left(y_{1}, y_{2}\right) \in \mathbb{R}^{2}$. By the definition of $f_{1}$ and $f_{2}$, we get

$$
\begin{aligned}
0 \leq & F_{1}\left(z_{n}, y\right)+\left\langle f_{1}\left(z_{n}\right), y-z_{n}\right\rangle+\frac{1}{r}\left\langle y-z_{n}, z_{n}-x_{n}\right\rangle \\
= & -\left(z_{n}^{1}\right)^{2}-\left(z_{n}^{2}\right)^{2}+\left(y_{1}\right)^{2}+\left(y_{2}\right)^{2}+\frac{1}{5} z_{n}^{1}\left(-z_{n}^{1}+y_{1}\right)+\frac{1}{5} z_{n}^{2}\left(-z_{n}^{2}+y_{2}\right) \\
& +\left(y_{1}-z_{n}^{1}\right)\left(z_{n}^{1}-x_{n}^{1}\right)+\left(y_{2}-z_{n}^{2}\right)\left(z_{n}^{2}-x_{n}^{2}\right) \\
= & \left(\left(y_{1}\right)^{2}+\left(-x_{n}^{1}+\frac{6}{5} z_{n}^{1}\right) y_{1}+x_{n}^{1} z_{n}^{1}-\frac{11}{5}\left(z_{n}^{1}\right)^{2}\right) \\
& +\left(\left(y_{2}\right)^{2}+\left(-x_{n}^{2}+\frac{6}{5} z_{n}^{2}\right) y_{2}+x_{n}^{2} z_{n}^{2}-\frac{11}{5}\left(z_{n}^{2}\right)^{2}\right) \\
= & G_{1}\left(y_{1}\right)+G_{2}\left(y_{2}\right) .
\end{aligned}
$$

Let $G_{1}\left(y_{1}\right)=\left(y_{1}\right)^{2}+\left(-x_{n}^{1}+\frac{6}{5} z_{n}^{1}\right) y_{1}+x_{n}^{1} z_{n}^{1}-\frac{11}{5}\left(z_{n}^{1}\right)^{2}$ and $G_{2}\left(y_{2}\right)=\left(y_{2}\right)^{2}+\left(-x_{n}^{2}+\frac{6}{5} z_{n}^{2}\right) y_{2}+x_{n}^{2} z_{n}^{2}-$ $\frac{11}{5}\left(z_{n}^{2}\right)^{2} \cdot G_{1}\left(y_{1}\right)$ and $G_{2}\left(y_{2}\right)$ are quadratic functions with coefficients $a_{1}=1, b_{1}=-x_{n}^{1}+\frac{6}{5} z_{n}^{1}$, and $c_{1}=x_{n}^{1} z_{n}^{1}-\frac{11}{5}\left(z_{n}^{1}\right)^{2}$ of $G_{1}\left(y_{1}\right)$ and coefficients $a_{2}=1, b_{2}=-x_{n}^{2}+\frac{6}{5} z_{n}^{2}$, and $c_{2}=x_{n}^{2} z_{n}^{2}-$ $\frac{11}{5}\left(z_{n}^{2}\right)^{2}$ of $G_{2}\left(y_{2}\right)$, respectively. Determine the discriminant $\Delta_{1}$ of $G_{1}$ as follows:

$$
\begin{aligned}
\Delta_{1} & =b_{1}^{2}-4 a_{1} c_{1} \\
& =\left(-x_{n}^{1}+\frac{6}{5} z_{n}^{1}\right)^{2}-4(1)\left(x_{n}^{1} z_{n}^{1}-\frac{11}{5}\left(z_{n}^{1}\right)^{2}\right)=\frac{1}{25}\left(5 x_{n}^{1}-16 z_{n}^{1}\right)^{2} .
\end{aligned}
$$

We know that $G_{1}\left(y_{1}\right) \geq 0, \forall y \in \mathbb{R}$. If it has most one solution in $\mathbb{R}$, then $\Delta_{1} \leq 0$, so we obtain $z_{n}^{1}=\frac{5 x_{n}^{1}}{16}$. Next, we determine the discriminant $\Delta_{2}$ of $G_{2}$ by using the same method as above, we obtain $z_{n}^{2}=\frac{5 x_{n}^{2}}{16}$. That is $T_{r}^{F_{1}}\left(I-r f_{1}\right) z_{n}=\left(\frac{5 x_{n}^{1}}{16}, \frac{5 x_{n}^{2}}{16}\right)$. After that, we find the solution of $u_{n}=\left(u_{n}^{1}, u_{n}^{2}\right)$ in this inequality $0 \leq F_{2}\left(u_{n}, y\right)+\left\langle f_{2}\left(u_{n}\right), y-u_{n}\right\rangle+\frac{1}{s}\left\langle y-u_{n}, u_{n}-x_{n}\right\rangle$. By using the same method as $z_{n}=\left(z_{n}^{1}, z_{n}^{2}\right)$, we obtain

$$
u_{n}=\left(u_{n}^{1}, u_{n}^{2}\right)=\left(\frac{7 x_{n}^{1}}{51}, \frac{7 x_{n}^{2}}{51}\right)
$$

That is, $T_{s}^{F_{2}}\left(I-s f_{2}\right) u_{n}=\left(\frac{7 x_{n}^{1}}{51}, \frac{7 x_{n}^{2}}{51}\right)$.

Let $x_{1}=\left(x_{1}^{1}, x_{1}^{2}\right)$ and $u=\left(u_{1}, u_{2}\right) \in \mathbb{R}^{2}$. The sequences $\left\{x_{n}\right\},\left\{y_{n}\right\}$ and $\left\{u_{n}\right\}$ are generated by (3.6), where $k_{i}=\frac{6}{7^{i}}+\frac{1}{N 7^{N}}, d_{1}=1, d_{2}=1, a=0.5, \alpha_{n}=\frac{1}{2 n}, \beta_{n}=\frac{7 n-4}{12 n}, \gamma_{n}=\frac{5 n-2}{12 n}$ and $\lambda_{n}=\frac{1}{2 n^{2}}$ for all $n \in \mathbb{N}$. Since $L=5$, we choose $\gamma=0.1$. From the definition of $D_{1}, D_{2}, T_{i}, F_{1}, F_{2}, f_{1}$ and $f_{2}$, we have $V I\left(C, D_{1}\right) \cap V I\left(C, D_{2}\right) \cap \bigcap_{i=1}^{N} F\left(T_{i}\right) \cap \Omega=\{0\}$. From Theorem 3.4, we can conclude that the sequences $\left\{x_{n}\right\},\left\{y_{n}\right\}$ and $\left\{u_{n}\right\}$ converge strongly to 0 . We can rewrite (3.6) as follows:

$$
\left\{\begin{array}{l}
u_{n}=T_{1}^{F_{1}}\left(I-f_{1}\right)\left(x_{n}+0.1 A^{*}\left(T_{0.5}^{F_{2}}\left(I-0.5 f_{2}\right)-I\right) A x_{n}\right) \\
y_{n}=\left(I-D_{1}\right)\left(0.5 u_{n}+0.5\left(I-D_{2}\right) u_{n}\right) \\
x_{n+1}=\frac{1}{2 n} u+\frac{7 n-4}{12 n} x_{n}+\frac{5 n-2}{12 n}\left(y_{n}-\left(\left(\frac{1}{2 n^{2}}\right)\left(\sum_{i=1}^{N}\left(\frac{6}{7^{i}}+\frac{1}{N 7^{N}}\right)\left(y_{n}-T_{i} y_{n}\right)\right)\right)\right)
\end{array}\right.
$$

for all $n \geq 1$, where $x_{n}=\left(x_{n}^{1}, x_{n}^{2}\right), y_{n}=\left(y_{n}^{1}, y_{n}^{2}\right)$ and $u_{n}=\left(u_{n}^{1}, u_{n}^{2}\right)$. 
Table 1 The values of $\left\{x_{n}\right\},\left\{y_{n}\right\}$ and $\left\{u_{n}\right\}$ where $u=(5,-5), x_{1}=(5,-5)$ and $n=30$

\begin{tabular}{|c|c|c|c|c|c|c|}
\hline \multirow[b]{2}{*}{$n$} & \multicolumn{3}{|l|}{$N=1$} & \multicolumn{3}{|l|}{$N=20$} \\
\hline & $x_{n}=\left(x_{n}^{1}, x_{n}^{2}\right)$ & $y_{n}=\left(y_{n}^{1}, y_{n}^{2}\right)$ & $u_{n}=\left(u_{n}^{1}, u_{n}^{2}\right)$ & $x_{n}=\left(x_{n}^{1}, x_{n}^{2}\right)$ & $y_{n}=\left(y_{n}^{1}, y_{n}^{2}\right)$ & $u_{n}=\left(u_{n}^{1}, u_{n}^{2}\right)$ \\
\hline 1 & $(5.6$ & $(0.5$ & $(0.8$ & 00) & $(0$. & $3885)$ \\
\hline 2 & (3.871 & $(0.4300,-0.4794)$ & $(0.6879$ & $(3.870$ & $(0.4298,-0.4792)$ & (0.6877, \\
\hline : & & & & & $\vdots$ & $\vdots$ \\
\hline 15 & $(0.5189,-0.5274)$ & $(0.0576,-0.0651)$ & $(0.0922,-0.0937)$ & $(0.5189,-0.5274)$ & $(0.0576,-0.0651)$ & $(0.0922,-0.0937)$ \\
\hline : & $\vdots$ & & & $\vdots$ & : & \\
\hline 29 & $(0.24 \varepsilon$ & $(0.02$ & $(0.04$ & $(0.2$ & $(0.0$ & $(0.04$ \\
\hline 30 & $(0.2397,-0.2432)$ & $(0.0266,-0.0300)$ & $(0.0426,-0.0432)$ & $(0.2397,-0.2432)$ & $(0.0266,-0.0300)$ & $(0.0426,-0.0432)$ \\
\hline
\end{tabular}

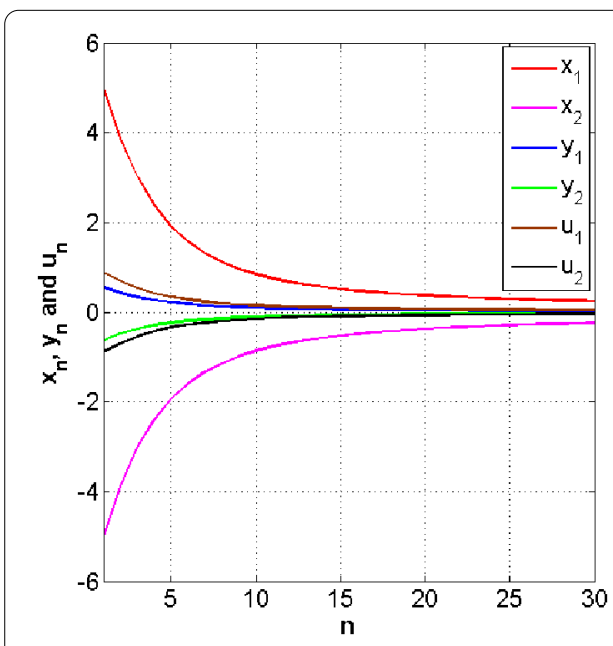

(a) $N=1$

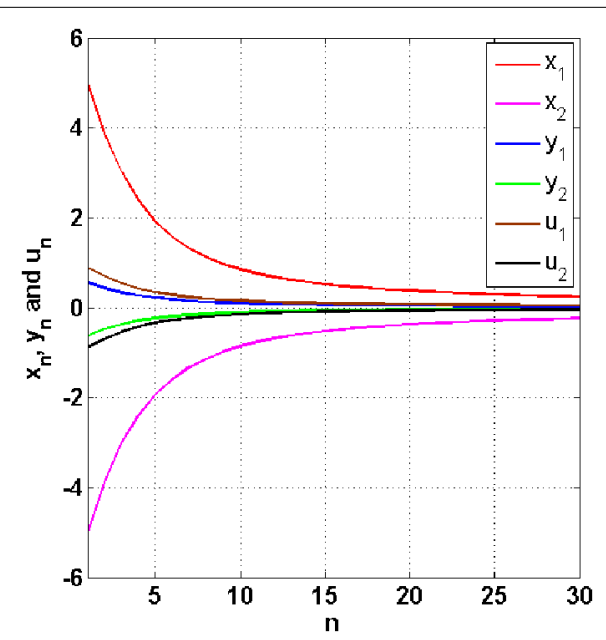

(b) $N=20$

Figure 1 The convergence comparison with different values $N$

Table 1 shows the values of sequences $\left\{x_{n}\right\},\left\{y_{n}\right\}$ and $\left\{u_{n}\right\}$ where $u=(5,-5), x_{1}=(5,-5)$ and $n=30$.

\section{Conclusion}

1. Example 5.1 is an example in infinite dimensional Hilbert space for supporting Theorem 3.4

2. Table 1 and Fig. 1 in Example 5.2 show that the sequences $\left\{x_{n}\right\},\left\{y_{n}\right\}$ and $\left\{u_{n}\right\}$ converge to 0 , where $\{0\}=V I\left(C, D_{1}\right) \cap V I\left(C, D_{2}\right) \cap \bigcap_{i=1}^{N} F\left(T_{i}\right) \cap \Omega$.

3. Theorem 3.4 guarantees the convergence of $\left\{x_{n}\right\},\left\{y_{n}\right\}$ and $\left\{u_{n}\right\}$ in Example 5.1 and Example 5.2.

4. By using the concept of Picard iteration, Wang [13] defined the iterative scheme $\left\{x_{n}\right\}$ for solving SCFPP as follows:

$$
\begin{aligned}
x_{n+1} & =x_{n}-\rho_{n}\left((I-U) x_{n}+A^{*}(I-T) A x_{n}\right) \\
& =\left(I-\rho_{n}\left((I-U)+A^{*}(I-T) A\right)\right) x_{n},
\end{aligned}
$$


where $\rho_{n}$ is according to (1.4) and $U$ and $T$ are firmly quasi-nonexpansive mappings. Then the sequence $\left\{x_{n}\right\}$ converges weakly to $z$, where $z=\lim _{n \rightarrow \infty} P_{\Phi} x_{n}$. In Theorem 3.4, we use the concept of Halpern iteration and suitable conditions of the parameters $d_{1}, d_{2}, r, s, a, \gamma, L,\left\{\alpha_{n}\right\},\left\{\beta_{n}\right\}$ and $\left\{\gamma_{n}\right\}$, the sequence $\left\{x_{n}\right\}$ defined by (3.6) converges strongly to $z=P_{\mathcal{F}} u$, which is a different method from (6.1).

\section{Acknowledgements}

This paper was supported by the Royal Golden Jubilee (RGJ) Ph.D. Programme, the Thailand Research Fund (TRF), under Grant No. PHD/0082/2558 and the Research and Innovation Services of King Mongkut's Institute of Technology Ladkrabang.

\section{Competing interests}

The authors declare that they have no competing interests.

Authors' contributions

The two authors contributed equally to the writing of this paper. Both authors read and approved the final manuscript.

\section{Publisher's Note}

Springer Nature remains neutral with regard to jurisdictional claims in published maps and institutional affiliations.

Received: 2 January 2018 Accepted: 11 May 2018 Published online: 22 May 2018

\section{References}

1. Goebel, K., Reich, S.: Uniform Convexity. Hyperbolic Geometry, and Nonexpansive Mappings. Marcel Dekker, New York (1984)

2. Ceng, L.C., Wang, C.Y., Yao, J.C.: Strong convergence theorems by a relaxed extragradient method for a general system of variational inequalities. Math. Methods Oper. Res. 67, 375-390 (2008)

3. Kangtunyakarn, A.: An iterative algorithm to approximate a common element of the set of common fixed points for a finite family of strict pseudo-contractions and of the set of solutions for a modified system of variational inequalities. Fixed Point Theory Appl. 2013, 143 (2013)

4. Blum, E., Oettli, W.: From optimization and variational inequalities to equilibrium problems. Math. Stud. 63(14), $123-145$ (1994)

5. Kim, J.K., Buong, N.: A new iterative method for equilibrium problems and fixed point problems for infinite family of nonself strictly pseudocontractive mappings. Fixed Point Theory Appl. 2013, 286 (2013)

6. Takahashi, W., Wong, N.C., Yao, J.C.: Iterative common solutions for monotone inclusion problems, fixed point problems and equilibrium problems. Fixed Point Theory Appl. 2012, 181 (2012)

7. Kassay, G., Reich, S., Sabach, S.: Iterative methods for solving systems of variational inequalities in reflexive Banach spaces. SIAM J. Optim. 21, 1319-1344 (2011)

8. Reich, S., Sabach, S.: Three strong convergence theorems regarding iterative methods for solving equilibrium problems in reflexive Banach spaces. Contemp. Math. 568, 225-240 (2012)

9. Censor, Y.: A multiprojection algorithm using Bregman projections in a product space. Numer. Algorithms 8, 221-239 (1994)

10. Byrne, C.: Iterative oblique projection onto convex sets and the split feasibility problem. Inverse Probl. 18,441-453 (2002)

11. Censor, Y., Gibali, A., Reich, S.: Algorithms for the split variational inequality problem. Numer. Algorithms 59, 301-323 (2012)

12. Moudafi, A.: Split monotone variational inclusions. J. Optim. Theory Appl. 150, 275-283 (2011)

13. Wang, F: A new method for split common fixed-point problem without priori knowledge of operator norms. J. Fixed Point Theory Appl. 19, 2427-2536 (2017)

14. Gibali, A.: A new split inverse problem and application to least intensity feasible solutions. Pure Appl. Funct. Anal. 2(2), 243-258 (2017)

15. Censor, Y., Bortfeld, T., Martin, B., Trofimov, A.: A unified approach for inversion problems in intensity modulated radiation therapy. Phys. Med. Biol. 51, 2353-2365 (2006)

16. Combettes, P.L.: The convex feasibility problem in image recovery. Adv. Imaging Electron Phys. 95, 155-453 (1996)

17. Byrne, C.: Iterative oblique projection onto convex sets and the split feasibility problem. Inverse Probl. 18, 441-453 (2002)

18. Tian, M. Jin, X.: A general iterative method for quasi-nonexpansive mappings in Hilbert space. J. Inequal. Appl. 2012, 38 (2012)

19. Reich, S.: A limit theorem for projections. Linear Multilinear Algebra 13, 281-290 (1983)

20. Opial, Z:: Weak convergence of the sequence of successive approximation of nonexpansive mappings. Bull. Am. Math. Soc. 73, 591-597 (1967)

21. Combettes, P.L., Hirstoaga, S.A.: Equilibrium programming in Hilbert spaces. J. Nonlinear Convex Anal. 6(1), 117-136 (2005)

22. Takahashi, W: Nonlinear Functional Analysis. Yokohama Publishers, Yokohama (2000)

23. Xu, H.K.: An iterative approach to quadric optimization. J. Optim. Theory Appl. 116, 659-678 (2003)

24. Cheawchan, K., Kangtunyakarn, A.: Approximation method for fixed points of nonlinear mapping and variational inequalities with application. Thai J. Math. 13(3), 653-672 (2015) 
25. Kohsaka, F., Takahashi, W.: Fixed point theorems for a class of nonlinear mappings related to maximal monotone operators in Banach spaces. Arch. Math. 91, 166-177 (2008)

26. lemoto, S., Takahashi, W: Approximating common fixed points of nonexpansive mappings and nonspreading mappings in a Hilbert space. Nonlinear Anal. 71, 2082-2089 (2009)

27. Kangtunyakarn, A., Suantai, S.: Hybrid iterative scheme for generalized equilibrium problems and fixed point problems of finite family of nonexpansive mappings. Nonlinear Anal. Hybrid Syst. 3, 296-309 (2009)

28. Kangtunyakarn, A.: Strong convergence of the hybrid method for a finite family of nonspreading mappings and variational inequality problem. Fixed Point Theory Appl. 2012, 188 (2012)

Submit your manuscript to a SpringerOpen ${ }^{\circ}$ journal and benefit from:

- Convenient online submission

- Rigorous peer review

Open access: articles freely available online

- High visibility within the field

- Retaining the copyright to your article

Submit your next manuscript at $\gg$ springeropen.com 